\title{
3-羟基氧化吲哚的不对称合成：金属催化和有机催化
}

\author{
刘运林 ${ }^{a}$ 朱 锋 ${ }^{a}$ 王翠红 ${ }^{a}$ 周 剑*,a \\ ( ${ }^{a}$ 华东师范大学化学系 绿色化学与化工过程绿色化上海市重点实验室 上海 200062)
}

\begin{abstract}
摘要 3-取代的 3-着基氧化吲哚广泛存在于天然产物和药物活性分子中, 是一类受到合成化学家广泛关注的优势骨 架. 近年来, 围绕这一骨架的不对称催化合成取得了很大的进展. 本综述旨在介绍几类重要的 3-羊基氧化吲哚结构单 元的不对称催化合成方法, 藉此讨论有机催化和金属催化这两种不同催化模式各自的一些特点和优势之处.
\end{abstract}

关键词 3-取代的 3-羟基氧化吲哚; 不对称催化; 金属催化; 有机催化

\section{Metal Catalysis versus Organocatalysis in the Catalytic Asymmetric Synthesis of 3-Hydroxyoxindole}

\author{
Liu, Yunlin $^{a} \quad$ Zhu, Feng $^{a} \quad$ Wang, Cuihong $^{, a} \quad$ Zhou, Jian $^{*, a}$ \\ ( ${ }^{a}$ Shanghai Key Laboratory of Green Chemistry and Chemical Processes, Department of Chemistry, \\ East China Normal University, Shanghai 200062)
}

\begin{abstract}
Substituted 3-hydroxyoxindoles are widely encountered in a large number of natural products, drugs and pharmaceutically active compounds. The catalytic asymmetric construction of this privileged skeleton has attracted great attention from synthetic chemistry, and much progress has been made in recent years. This review summarizes the advances in the catalytic enantioselective synthesis of four important types of subunits of 3-substituted 3-hydroxyoxindoles, and focuses on the discussion of the differences and advantages of metal catalysis and organocatalysis.
\end{abstract}

Keywords 3-substituted-3-hydroxyoxindole; asymmetric catalysis; metal catalysis; organocatalysis

3-取代的3-着基氧化吲哚是一类非常重要的手性砌 块, 广泛存在于一些具有强烈生物活性的天然产物中 (Scheme 1) ${ }^{[1 \mathrm{a} \sim 1 \mathrm{f}]}$. 例如: maremycins $A \sim B(\mathbf{1})$ 对人类白 血病细胞 K562 具有抗细胞毒性 ${ }^{[1 \mathrm{~g}]}$, convolutamydine A 具有潜在的抗肿瘤活性 ${ }^{[1 \mathrm{~h}, 1 \mathrm{i}]}, \mathrm{CPC}-1$ (4) 则具有抗菌活 性 $^{[1 \mathrm{j}]}$. 同时, 这一结构单元也广泛存在于药物和药物活 性分子中, 如药物分子 TMC-95A (6) 是一类强效的蛋白 酶体抑制剂 ${ }^{[1 k]}$. 结构与活性之间的关系研究表明, C(3) 位的取代基对这类化合物的生物活性有着重要的影响. 例如, 由 Kamano 小组首先从佛罗里达州的海洋苔藓虫 Amathia convoluta 中分离到的天然产物 Convolutamydines $\mathrm{A} \sim \mathrm{E}(\mathbf{3})$ 的生物活性受 $\mathrm{C}(3)$ 位上的取代基的不同 而发生很大变化. 如在抑制人类早幼粒白血病细胞 HL-60 分化时, Convolutamydine A 的半抑制浓度 $\left(\mathrm{IC}_{50}\right)$
是 $0.1 \mu \mathrm{g} \cdot \mathrm{mL}^{-1}$, 而 Convolutamydine B 的半抑制浓度则 是 $12.5 \mu \mathrm{g} \cdot \mathrm{mL}^{-1}[\mathrm{lh}, 1 \mathrm{i}]$. 另外, $\mathrm{C}(3)$ 位的绝对构型也对这类 化合物的生物活性有着重要影响 ${ }^{[1 f]}$, 如口服非肽荷尔蒙 生长激素药物 SM-130686 (5), (S)构型的对映异构体与 $(R)$ 构型的半最大效应浓度 $\mathrm{EC}_{50}$ 差异达到 70 倍 ${ }^{[11]}$, 因此, 合成结构多样的 3-取代的 3-羟基氧化吲哚类手性化合 物库, 有助于进一步研究结构与活性之间的相互关系, 进而加快新药研发. 基于上述原因, 获得光学纯的 3-取 代的-3-差基氧化吲哚受到了合成化学及药物化学研究 者的广泛关注. 过去十年中, 利用手性试剂或手性辅基 的方法已经取得了很大成功 ${ }^{[2]}$, 但由于构建四取代碳手 性中心这一工作本身的挑战性, 导致相关不对称催化的 研究进展缓慢. 发展不对称催化的方法可以实现手性增 殖，从合成效率来考虑无疑更有优势. 可喜的是，随着

\footnotetext{
*E-mail: jzhou@chem.ecnu.edu.cn

Received March 24, 2013; revised April 13, 2013; published online April 24, 2013.

Project supported by the National Natural Science Foundation of China (Nos. 20902025, 21172075), the National Basic Research Program of China (973 Program, No. 2011CB808600), the Program for New Century Excellent Talents in University (No. NCET-11-0147), the Scholarship Award for Excellent Doctoral Student Granted by Ministry of Education (No. MXRZZ2012007).

国家自然科学基金(Nos. 20902025, 21172075)、国家重点基础研究发展计划(973 计划, No. 2011CB808600)、教育部新世纪优秀人才计划(No. NCET-11-0147)、教育部博士新人奖(No. MXRZZ2012007)资助项目.
} 
<smiles>[R]C1([C@@H](C)[C@@H]2NC(=O)[C@@H](CSC)NC2=O)C(=O)N(C)c2ccccc21</smiles>

$A: R=\cdots \cdot O H$

$\mathrm{B}: \mathrm{R}=-\mathrm{OH}$<smiles>CCN(CC)CCN1C(=O)[C@](O)(c2ccccc2Cl)c2c1cc(C(N)=O)cc2C(F)(F)F</smiles>

SM-130686 (5)

(S)-GHS-R EC $50=3 \mathrm{nmol} / \mathrm{L}$ $(R)-\mathrm{GHS}-\mathrm{R} \mathrm{EC} \mathrm{EC}_{50}=210 \mathrm{nmol} / \mathrm{L}$<smiles>CC(=S)NCC1(O)C(=O)Nc2ccccc21</smiles>

dioxibrassinine (2)<smiles>[R][C@@]1(O)C(=O)Nc2cc(Br)cc(Br)c21</smiles>

convolutamydine (3)

A: $\mathrm{R}=\mathrm{CH}_{2} \mathrm{COCH}_{3}$

B: $\mathrm{R}=\mathrm{CH}_{2} \mathrm{CH}_{2} \mathrm{Cl}$

C: $\mathrm{R}=\mathrm{CH}_{3}$

$\mathrm{D}: \mathrm{R}=\mathrm{CH}=\mathrm{CH}_{2}$

E: $\mathrm{R}=\mathrm{CH}_{2} \mathrm{CH}_{2} \mathrm{OH}$

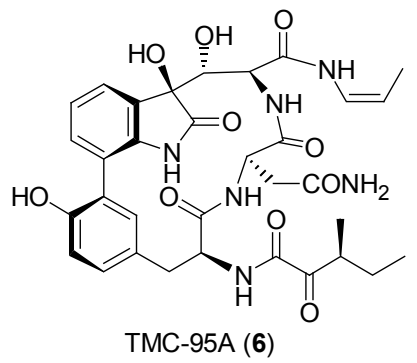

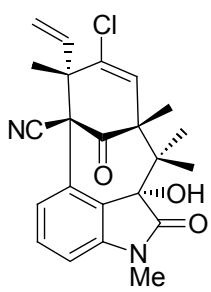

3-Hydroxy-N-methylwelwitindolinone $\mathrm{C}$ isonitrile (7)<smiles>CO[C@]12CCCN(C)[C@H]1c1ccc(Br)cc1N2C</smiles>

CPC-1 (4)

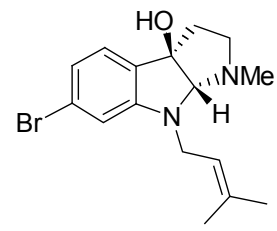

(-)-flustraminol B (8)

图 1 代表性的含 3-羟基氧化吲哚结构单元的天然产物和药物活性分子

Figure 1 Representative natural products and drugs that containing 3-hydroxyoxindole structural motif

手性金属催化和小分子催化这两种重要不对称催化方 法的不断发展和应用，一系列利用手性金属或有机小分 子催化剂的不对称催化新反应被开发出来, 成功地应用 于 3-取代的 3-羊基氧化吲哚的不对称构建, 具体策略可 分为以下几种: (a)亲核试剂对靛红的加成; (b) 3-羞基氧 化吲哚对亲电试剂的加成反应; (c)䤍酰胺的分子内环化 反应；(d) 3-烷基或芳基取代氧化吲哚的羟基化反应(图 2).

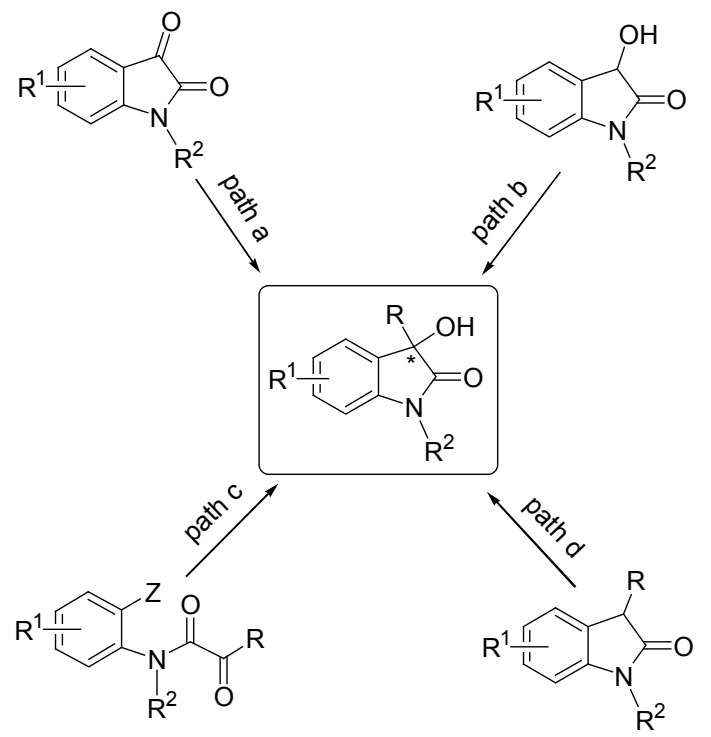

图 2 3-取代的-3-羟基氧化吲哚的合成策略

Figure 2 The synthetic strategies for 3-substituted-3-hydroxyoxindole

尽管目前已有几篇综述介绍了有关 3,3-双取代氧化
吲哚 ${ }^{[3 \mathrm{a} \sim 3 \mathrm{c}]}$ 、螺环氧化吲哚 ${ }^{[3 \mathrm{~d} \sim 3 \mathrm{f}]}$ 及 3-差基氧化吲哚 ${ }^{[3 \mathrm{~g}]}$ 的 不对称催化合成方法，但这些综述只是总结了已有的合 成方法，而针对不对称金属催化和有机催化这两种不同 的催化模式在构建 3-取代的-3-羟基氧化吲哚所表现出 来的特点和优势进行深度讨论的综述目前还没有.

众所周知, 自上世纪末以来, 在史一安 ${ }^{[4 a]}$, Jaco$\mathrm{bsen}^{[4 \mathrm{~b}, 4 \mathrm{c}]}, \mathrm{List}^{[\mathrm{dd}]}$, MacMillan ${ }^{[4 \mathrm{e}]}$ 等有机化学家的开创性工 作启发下，有机小分子催化取得了快速发展，成为继生 物催化和金属催化外的又一重要的不对称催化方法. 作 为两种化学催化方法, 不对称有机催化和金属催化各具 优点和局限性：有机小分子催化剂的稳定性较好，一种 催化剂可以具有多种催化模式, 对水和空气等杂质容忍 性较高，使用简便，无需担心产品的金属残留等优点， 但通常催化剂用量较大; 而金属催化往往活性较高，催 化剂用量较小，但也存在反应条件苛刻，金属离子残留 等问题. 总体上来说不对称金属催化和有机催化在开发 不对称新反应方面可实现优势互补.

本综述重点介绍 I IV 这 4 种重要的 3-取代的-3差基氧化吲哚结构单元(图 3)的不对称催化合成方法, 因为这些结构单元均是相关天然产物或药物分子的重 要合成中间体，同时具有金属催化和有机催化的合成方 法. 与已有综述不同的是，我们希望在本小组发展 3,3双取代氧化吲哚的高效合成方法的研究积累的基础 上 $^{[5]}$, 对近五年来, 这 4 种手性砌块的不对称金属催化 和有机催化的合成方法进行比较讨论，重点介绍不对称 金属催化和有机催化各自的特点、优势和使用范围, 为 从事氧化吲哚的不对称合成的研究人员提供参考. 
<smiles>[R]CCC1(O)C(=O)Nc2ccccc21</smiles>

I<smiles>[R]c1ccccc1C1(O)C(=O)Nc2ccccc21</smiles>

III<smiles>[R]C(=C)C1(O)C(=O)Nc2ccc([R])cc21</smiles>

II<smiles>[R]c1ccc2c(c1)C(O)([Al])C(=O)N2</smiles>

IV
图 3 四个典型的 3-羟基氧化吲哚砌块

Figure 3 Four classic building blocks of 3-hydroxyoxidoles

\section{3-着基-氧化吲哚手性砌块 I 的构建}

3-羟基氧化吲哚结构单元 $\mathbf{I}$ 是一类非常有用的合成 砌块, 可用于 convolutamydines $\mathrm{A} \sim \mathrm{E}, \mathrm{CPC}-1$ 等天然产 物及其类似物的合成. 自 2005 年以来，这一手性砌块的 不对称催化合成已有不少报道, 主要分为醛或酮与靛红 的直接 aldol 反应, 以及烯醇等当体与靛红的间接 aldol 反应, 如图 4 所示.
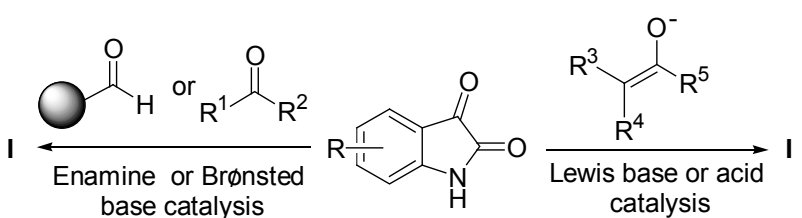

图 4 3-羟基氧化吲哚 $\mathbf{I}$ 的合成策略

Figure 4 The synthetic strategies of 3-hydroxyoxindole I

利用羰基化合物与靛红的 aldol 反应是构建 3-差基 氧化吲哚砌块 $\mathbf{I}$ 的最直接和经济的方法, 因为靛红和羰 基化合物均简单易得. 此外, 靛红是一类活性很高的 1,2-二羰基化合物, 其特点在于: (1)靛红为平面结构, 其 C3 位酮羰基受邻位酰胺基团的拉电子作用及芳环的影 响, 比图 5 所示的其他非环状 1,2-二羰基化合物的酮羰 基更容易接受亲核试剂的进攻; (2)由于没有 $\alpha$-位活泼 氢, 靛红不会发生自身 aldol 反应. 因此, 基于靛红的不 对称催化反应近年来得到广泛发展.

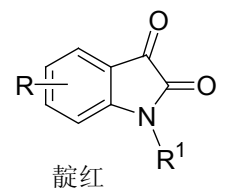

刚性平面结构 活性很高

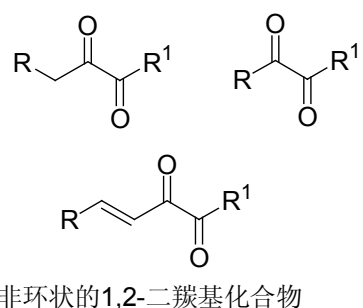

非环状的1,2-二羰基化合物
图 5 常见的 1,2-二羰基化合物

Figure 5 The common 1,2-dicarbonyl compounds

\section{1 烯胺催化的醛或活泼酮与靛红的直接 aldol 反应}

烯胺催化 ${ }^{[6]}$ 是指含 $\alpha$ 位氢原子的羰基化合物在少量 布朗斯特酸的助催化下与一级或者二级胺形成烯胺, 增 强 $\alpha$ 位的亲核能力, 进而与另一亲电试剂如羰基化合物 等发生 aldol 反应, 如图 6 所示.

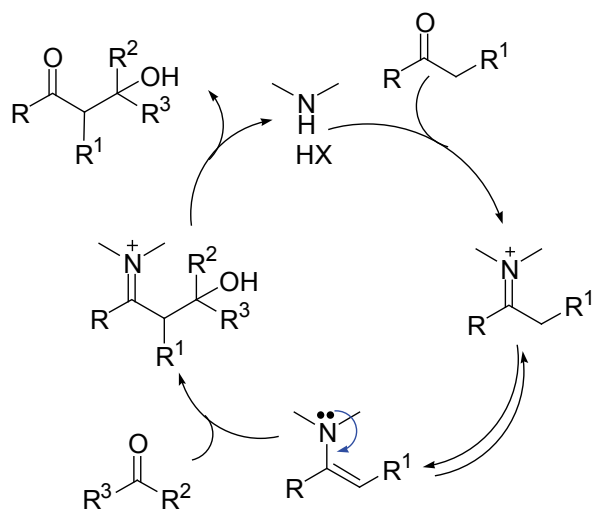

图 6 烯胺催化的 aldol 反应

Figure 6 Enamine catalyzed aldol reaction

自 List 等 ${ }^{[4 \mathrm{~d}]}$ 的开拓性工作之后，利用手性一级或者 二级胺来实现的不对称分子间 aldol 反应得到了广泛发 展，但直到 2005 年，才由 Tomasini 小组 ${ }^{[7 \mathrm{a}]}$ 首次实现了丙 酮与靛红的不对称 aldol 反应. 在脯氨酸衍生的二肽催 化剂 10a 作用下，反应以优秀的产率( $90 \%$ > >9\%)和中 等的对映选择性(73\% 77\% ee) 得到目标产物. 作者还 利用这一方法以 $68 \%$ 的 $e e$ 值实现了天然产物 $(R)$-convolutamydine $\mathrm{A}$ 的不对称全合成 ${ }^{[7 \mathrm{~b}]}$. 产物只需要 一次简单的重结晶, 就可把 $e e$ 值提高到 97\% (图 7). 虽 然此方法的底物普适性不好，但这是烯胺催化在这一价 值较高的手性砌块中的首次合成应用.

在此工作基础上，包括肖文精在内的多个小组尝试 优化手性胺催化剂来改进该反应的选择性和底物适用 范围 ${ }^{[7 \mathrm{c} \sim 7 \mathrm{i}]}$, 最好的结果由 Nakamura 等 ${ }^{[7 \mathrm{i}]}$ 报道. 他们利 用 $10 \mathrm{~b}$ 为催化剂, 成功实现了丙酮与靛红直接的高对映 选择性的 aldol 反应, ee 值高达 97\% (图 7).

此外, Singh 等 ${ }^{[8]}$ 利用该策略首次实现了环己酮与靛 红的高对映选择性 aldol 反应. 随后，他们又利用辛可宁 衍生的一级胺催化剂 14 实现了苯乙酮与靛红的高对映 选择性 aldol 反应，但是由于苯乙酮的活性较低，反应时 间较长 ${ }^{[9]}$ (图 8). 通过以上二个例子可以看出, 对于活性 较低及位阻相对较大的酮, 手性一级胺才能更为有效的 促进反应的顺利进行, 可能是因为一级胺较二级胺更容 易形成烯胺.

与酮羰基相比，醛羊基活性更高，在进行交叉的不 对称 aldol 反应时很容易发生自身的 aldol 反应，因此醛 与靛红的 aldol 反应更具挑战性. 2009 年, Nakamura 等 ${ }^{[10]}$ 

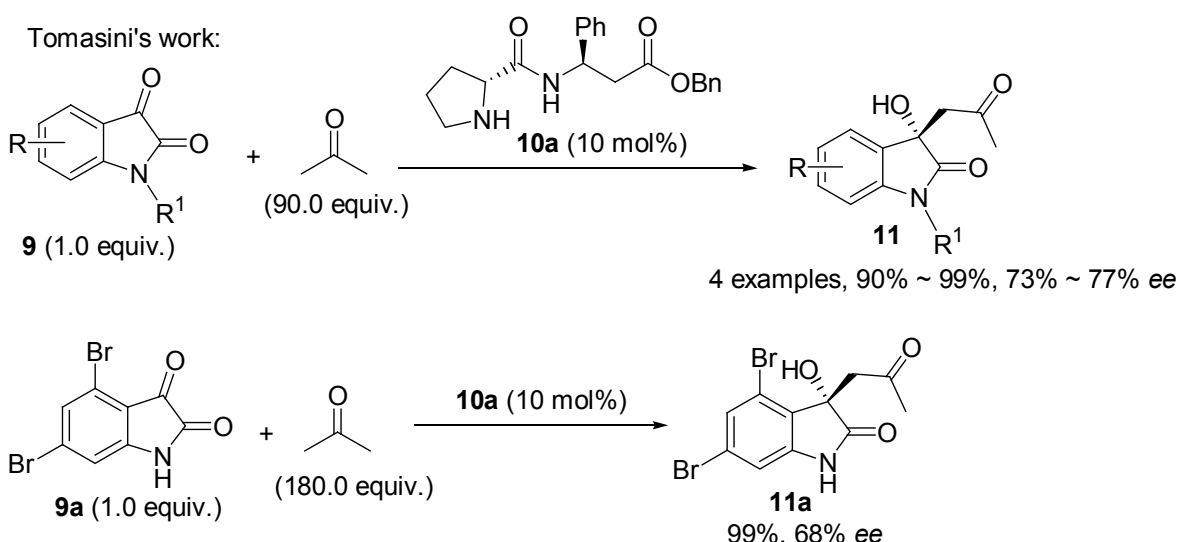

$50 \%, 97 \%$ ee after one crystallization<smiles>[R]c1ccc2c(c1)NC(=O)C2=O</smiles>

9 (1.0 equiv.)

$\mathrm{R}=\mathrm{H}$, 4-Me, 6-Me, halogen

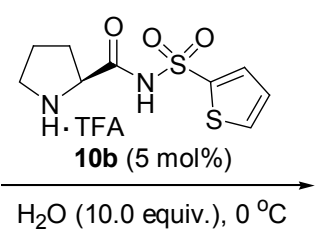

7 examples, $59 \%$ 99\%, $77 \%$ 97\% ee

图 7 靛红 9 与丙酮的 aldol 反应

Figure 7 The aldol reaction of isatins 9 and acetone

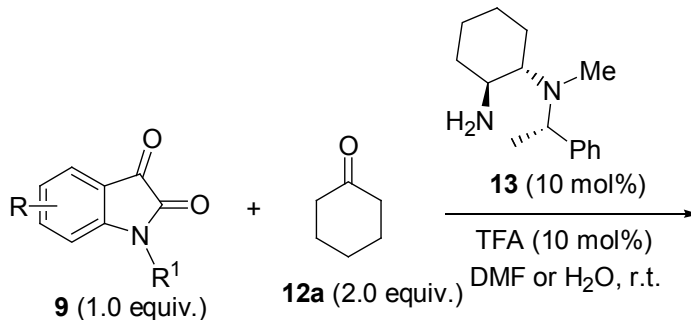

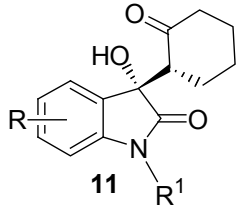

6 examples $83 \% \sim 92 \%$ yield $92: 8 \sim 99: 1 d r, 60 \% \sim 99 \%$ ee<smiles>O=C1C(=[OH+])Nc2ccccc21</smiles><smiles>CC(=O)O[Na]</smiles>
12b (20.0 equiv.) $9 b$ ( 1.0 equiv.)

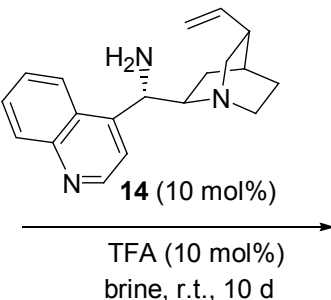<smiles>CN1C(=O)[C@](O)(CC(=O)c2ccccc2)c2ccccc21</smiles>

11b $\mathrm{H}$

$59 \%, 90 \%$ ee

图 8 苯乙酮、环己酮与青定红的 aldol 反应

Figure 8 The aldol reaction of cyclohexanone and acetophenone with isatins

首次报道了直链的醛与靛红的高对映选择性的 aldol 反 应，在催化剂 10b 的作用下，以高达 99\%的产率及 $98 \%$ 的对映选择性得到相应的目标产物 11. 而靛红和乙醛的 高选择性反应也成功地应用到天然产物 $(R)$-convolutamydines B 和 $\mathrm{E}$ 的全合成中(图 9).
随后，王卫小组发现在催化剂 $\mathbf{1 0 c}$ 的作用下 ${ }^{[11]}$, 增大醛 的用量为静红的五倍, 支链及直链的醛都可以方便的与 靛红顺利的进行反应，以中等到优秀的产率(46\% 92\%)及对映选择性(49\% 93\%)得到相应的产物 11. 这 一反应大大拓展了底物的范围(图 9). 

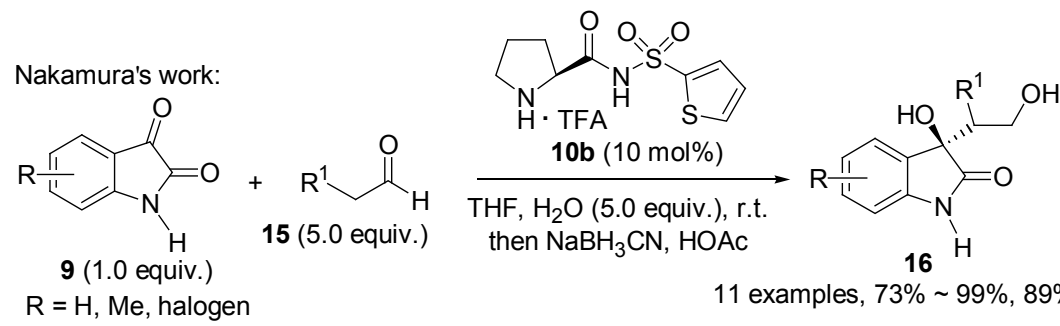

11 examples, $73 \% \sim 99 \%$, $89 \% \sim 98 \%$ ee

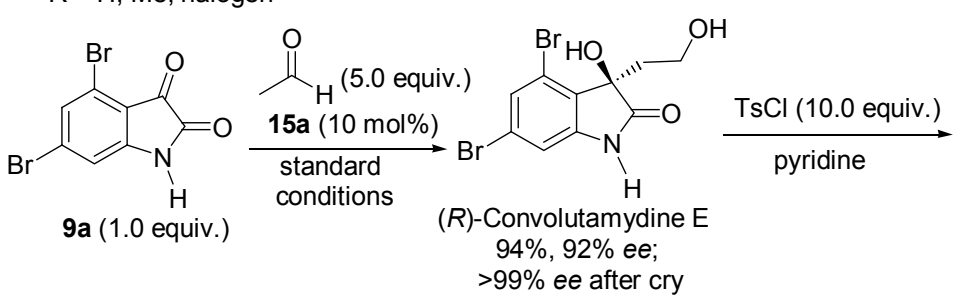<smiles>O=C1N(C([18OH])[18OH])c2cc(Br)cc(Br)c2[C@]1(O)CCCl</smiles>

Wang's work:<smiles>[R]#CC1=CC=[R]=CN1C([NH3+])=O</smiles><smiles>[R]C([R])C(=O)O</smiles>

9 (1.0 equiv.) 15 (5.0 equiv.)

$\mathrm{R}=\mathrm{H}$, Me, halogen; $\mathrm{R}^{2}=\mathrm{H}, \mathrm{Bn}$ $\mathrm{R}^{3}, \mathrm{R}^{4}=\mathrm{Me}, \mathrm{Me} ; \mathrm{H}, \mathrm{Me} ; \mathrm{H}, n-\mathrm{Bu}$

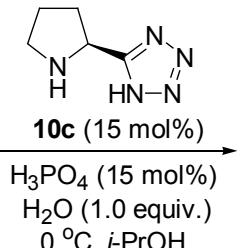

$0^{\circ} \mathrm{C}, i-\mathrm{PrOH}$

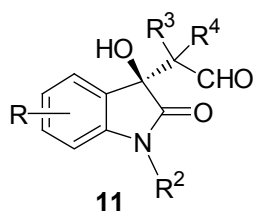

12 examples, $46 \% \sim 92 \%$

$49 \% \sim 93 \%$ ee, $1.4: 1 \sim 8: 1 d r$

图 9 靛红与醛的不对称 aldol 反应

Figure 9 The asymmetric aldol reaction of isatins and aldhydes

\section{2 布朗斯特碱催化的低活性酮与青定红直接 aldol 反应}

从上述例子可以发现，尽管利用手性一级或者二级 胺催化剂，通过烯胺催化的方式，可以促进易形成烯胺 的高活性的羰基化合物与靛红的反应来构建手性砌块 $\mathbf{I}$. 但是对于低活性的芳香酮、 $\alpha, \beta$-不饱和酮等与靛红的 aldol 反应, 则存在反应活性不足、时间较长等不足. 除
了 Singh 发展的一例苯乙醖对靛红的加成反应外，没有 其他报道. 这可能是因为: (1)非活化的芳香酮形成烯胺 相对不易; (2) $\alpha, \beta$-不饱和酮与手性胺催化剂可能会发生 Michael 加成等副反应; (3)即使形成烯胺，由于烯胺双键 与氮原子上的孤对电子之间轨道不共平面，使得轨道间 的重叠较少, 导致反应活性较低 ${ }^{[12]}$ (图 10). 因此如果
Enamine catalysis mechanism<smiles>O=C(CF)c1ccccc1</smiles>

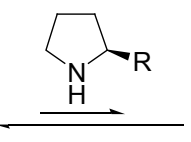

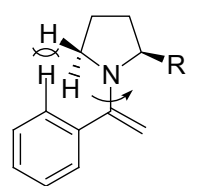
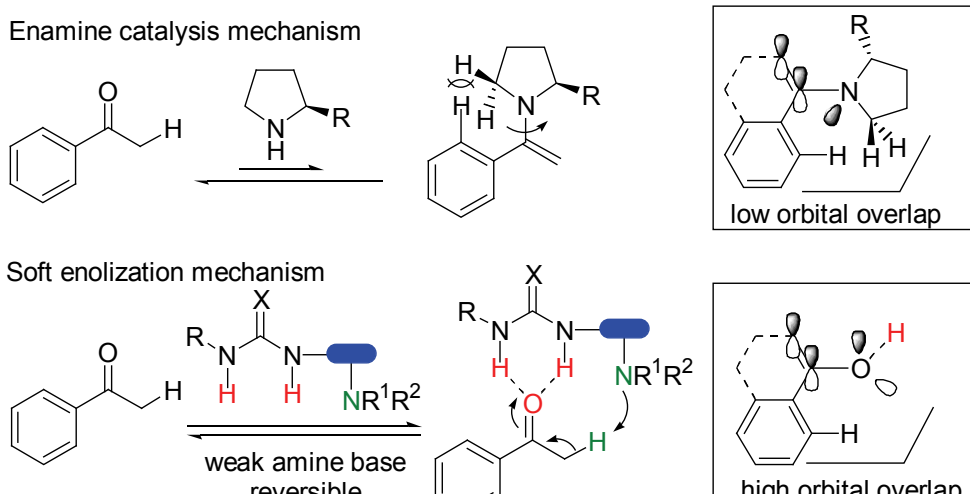

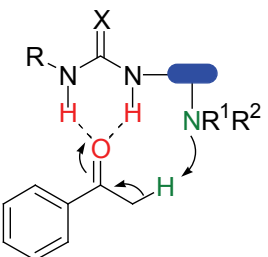

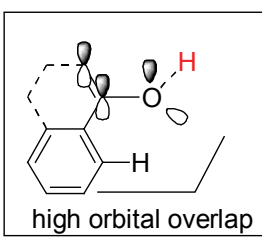

Hard enolization mechanism

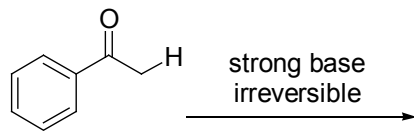<smiles>COc1ccccc1</smiles>

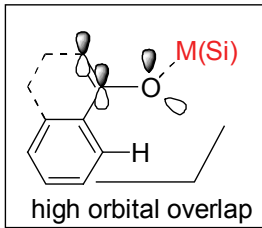

图 10 活性酮与非活性酮的反应机理

Figure 10 The reaction mechanism of activated and unactivated ketones 
要实现非活性芳香酮类化合物与靛红之间直接的 aldol 反应，则通常需要采用软烯醇化活化的机理 ${ }^{[9,13]}$, 即利 用相对较弱的胺碱原位擢氢活化, 同时利用手性催化剂 中的氢键活化靛红, 进而实现靛红与非活性醖直接的 aldol 反应.

2010 年, 赵丛贵小组 ${ }^{[14 a]}$ 率先对苯乙酮等非活性酤 与靛红的直接 aldol 反应进行了研究, 发现利用奎尼定 衍生的硫脲催化剂 17a 可以高对映选择性地实现这一反 应. 芳环上带不同取代基的靛红、苯乙酮及 $\alpha, \beta$-不饱和 酮化合物在该条件下均反应良好, 以中等到优秀的产率 和对映选择性得到目标产物. 作者推测反应的机理可能 是: 双功能奎尼定衍生的硫嫝催化剂 $17 \mathrm{a}$ 中的叔胺部分 篗取酮 $\alpha$ 位上的氢, 同时硫脲催化剂通过氢键活化青定红, 接受原位生成的烯醇负离子的亲核进攻, 进而生成相应 的目标产物. 总之对于不易形成烯胺的低活性酩的直接 的 aldol 反应, 布朗斯特碱催化提供了另外一种策略(图 11), 与烯胺催化形成优势互补. 随后, 王兴旺小组 ${ }^{[14 b]}$ 和蒋俊小组 ${ }^{[14 \mathrm{c}]}$ 利用相同的策略分别实现了 $\alpha, \beta$-不饱和 甲基醖及 1,3-二羰基化合物与靛红的不对称 aldol 反应.

\section{3 烯醇等当体与靛红间接的不对称 aldol 反应}

对于碱性较弱, 不易被布朗斯特碱原位篗氢活化的 非活性酮, 通常需要事先将此类酮制备成相应的烯醇硅 醚或烯醇金属盐. 采用硬烯醇化的策略实现此类非活性 酤与靛红之间间接的不对称 aldol 反应 ${ }^{[13 \mathrm{~d}]}$.

2011 年, Mikami 小组 ${ }^{[15]}$ 发展了首例手性二价钯络
合物催化的烯醇硅醚 19 与靛红的高对映选择性的 Mukaiyama aldol 反应. 有趣的是在反应过程中，作者发 现如果使用丙酮衍生的烯醇硅醚底物在标准条件下发 生的是高对映选择性的 ene 反应，而当底物是硫代烯醇 硅醚时发生的是 Mukaiyama aldol 反应，得到相应的 3羟基氧化吲哚类化合物 11. 作者认为靛红与手性钯的螯 合作用是反应取得高对映选择性及高反应活性的关键 (图 12).

除了使用需预先制备的烯醇硅醚来开发反应外， Shibata 小组 ${ }^{[16]}$ 采取了现场生成烯醇等当体的策略, 即 利用丙二酸单硫代酸酯 21 的现场脱羧的方式，结合使 用奎宁衍生的手性四方酸酰胺双功能催化剂 $17 \mathrm{~b}$, 高产 率高选择性地实现了对靛红的不对称脱酸加成反应(图 13).

在此基础上，作者利用这一方法以四步 $71 \%$ 的总产 率, $92 \%$ 的对映选择性实现了天然产物 flustraminol B 的 首次不对称全合成. 与 Mikami 小组工作相比, Shibata 小 组采用的硫代酸酯 21 对靛红的不对称脱酸反应，底物 的范围更广，操作更为简便.

向分子中引入二氟烷基已经成为药学研究中改善 化合物性质的一种有效手段，但利用不对称催化的方法 构建二氟烷基取代的手性中心的研究刚起步 ${ }^{[17]}$. 我们发 现二氟甲基取代的苯乙酩，并不能在 17a 的催化下与靛 红反应，因此利用相应的烯醇等当体二氟烯醇硅醚 19 来设计反应. 发现 19 可以在温和的条件下，被叔胺

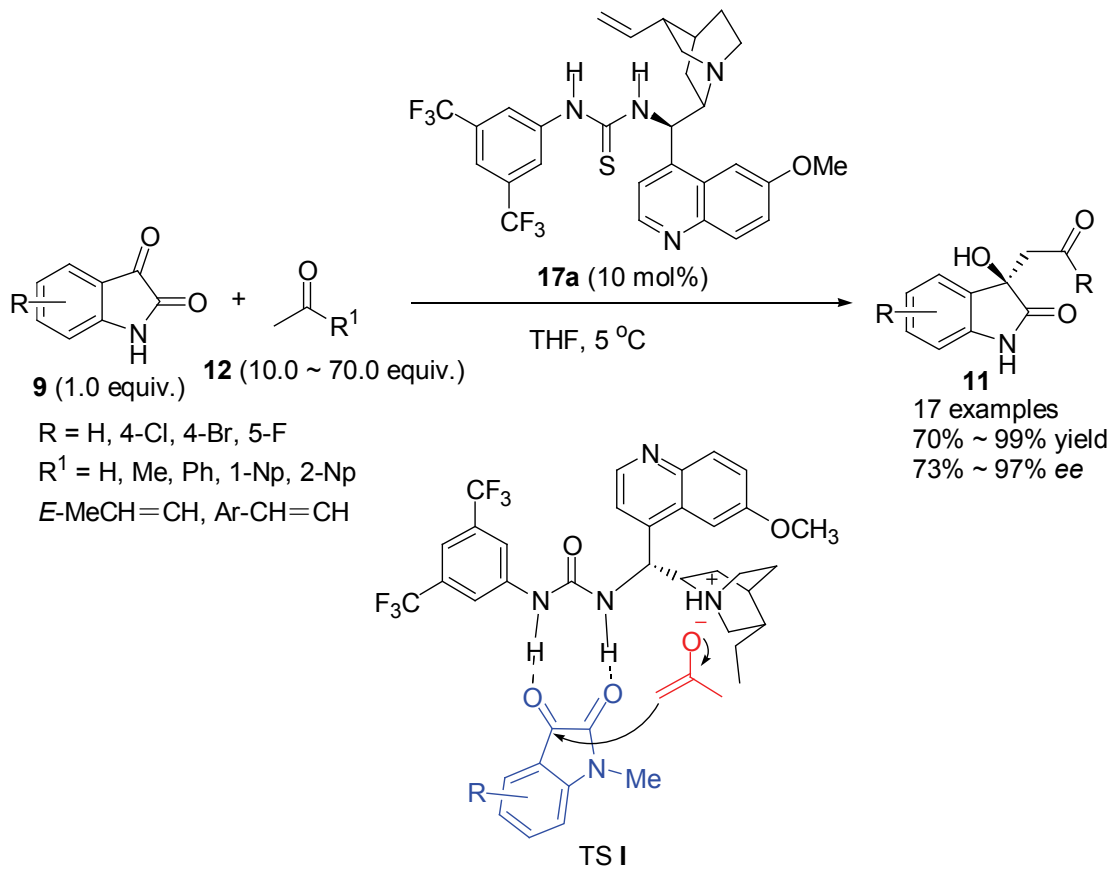

图 11 靛红与酮 12 的 aldol 反应

Figure 11 The aldol reaction of isatin and ketones 12 


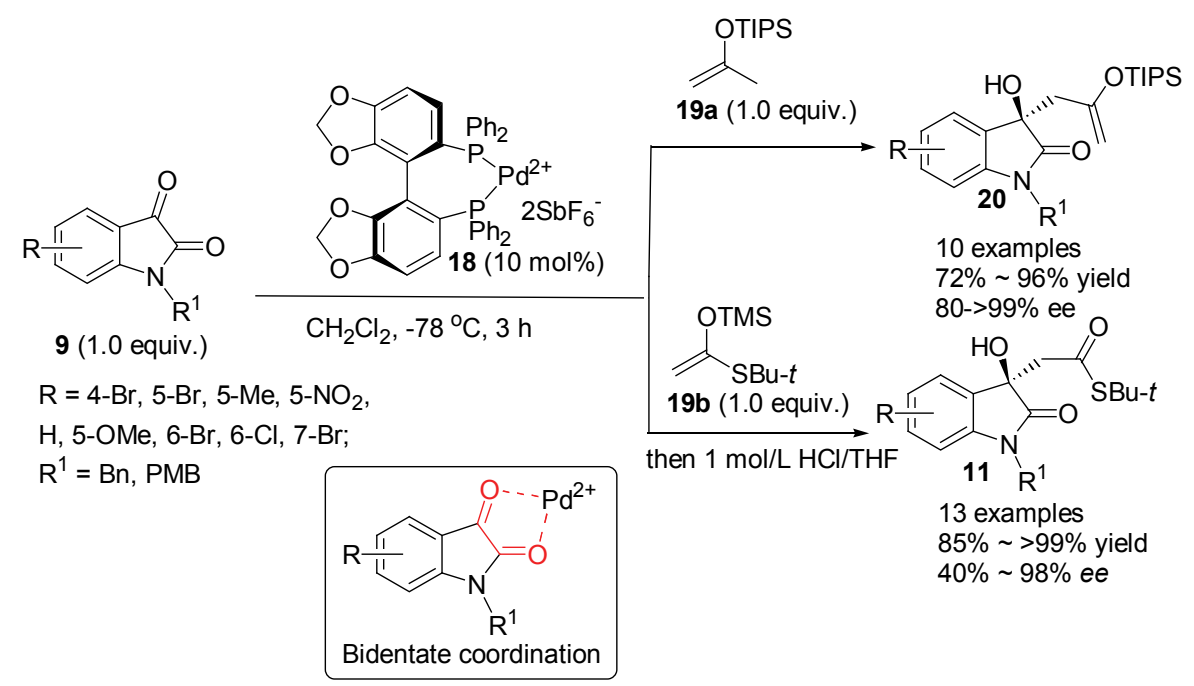

图 12 靛红与 19 的 Mukaiyama aldol 反应

Figure 12 The Mukaiyama aldol reaction of isatin and 19<smiles>O=C1Nc2ccccc2C1=O</smiles>

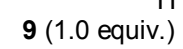<smiles>O=C(O)CC(=O)c1ccccc1</smiles>

21 (1.1 equiv.) cat.

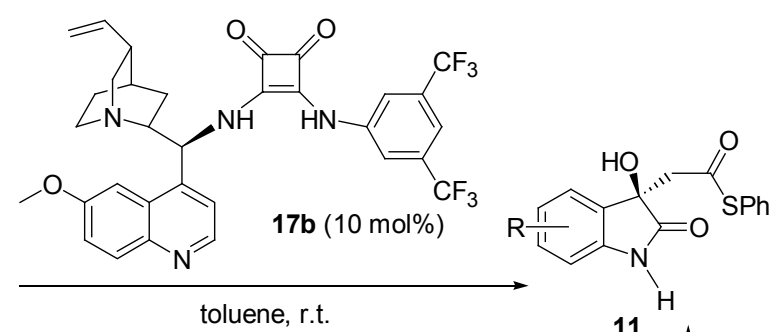

8 examples, $85 \% \sim>99 \%, 40 \% \sim 98 \%$ ee

11<smiles>CI</smiles>

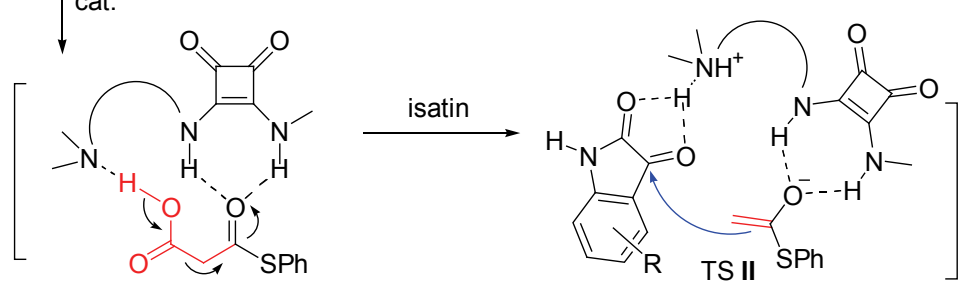

图 13 靛红与 21 的脱酸 aldol 反应

Figure 13 Decarboxylative aldol reaction of isatins and 21<smiles>COC(=O)C[C@]1(O)C(=O)N(CC=C(C)C)c2cc(Br)ccc21</smiles>

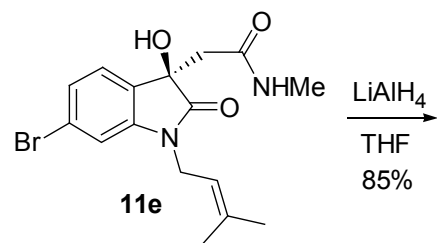

4 steps, $71 \%$ total yield, $92 \%$ ee

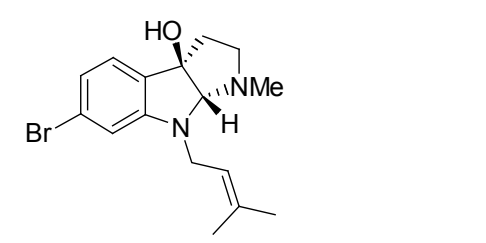

(-)-flustraminol B

图 14 Flustraminol B 的全合成

Figure 14 The total synthesis of flustraminol B 
Lewis 碱活化，进而与靛红反应. 在此发现基础上，我们 利用氢化奎宁衍生的嫝催化剂 $17 \mathrm{c}$ 实现了高产率和高对 映选择性的 19 与靛红的加成反应, 首次实现了二氟烷 基取代的 3-差基氧化吲哚化合物 11 的高选择性合成. 初步机理研究显示催化剂 $17 \mathrm{c}$ 的叔胺部分作为路易斯碱 可以高效活化二氟烯醇硅醚 19, 这是启动反应的关键.
这也是首例利用手性的中性叔胺催化的二氟烯醇硅醚 参与的不对称 Mukaiyama aldol 反应 ${ }^{[18]}$ (图 15).

利用该不对称催化的方法，我们实现了天然产物 Convolutamydine E 的二氟代类似物的首次全合成，而 其关键中间体 $11 \mathrm{f}$ 即是通过这一不对称方法来高选择性 构建(图 16).

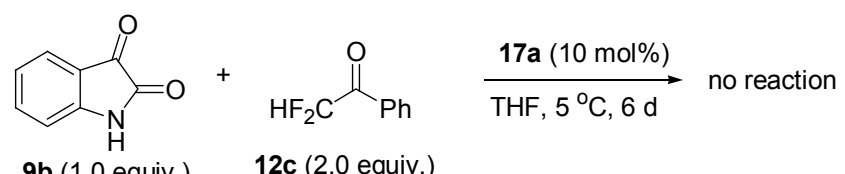

9b (1.0 equiv.)

12c (2.0 equiv.)

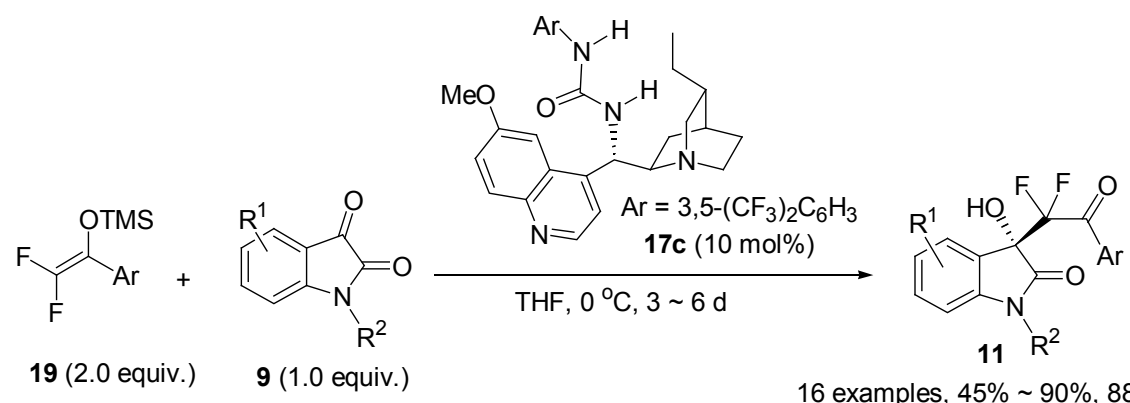

16 examples, $45 \%$ 90\%, 88\% 96\% ee

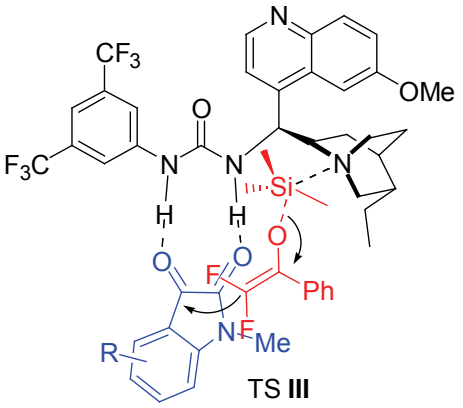

图 15 靛红与二氟烯醇硅醚 19 的 Mukaiyama aldol 反应

Figure 15 Mukaiyama aldol reaction of isatin and 19

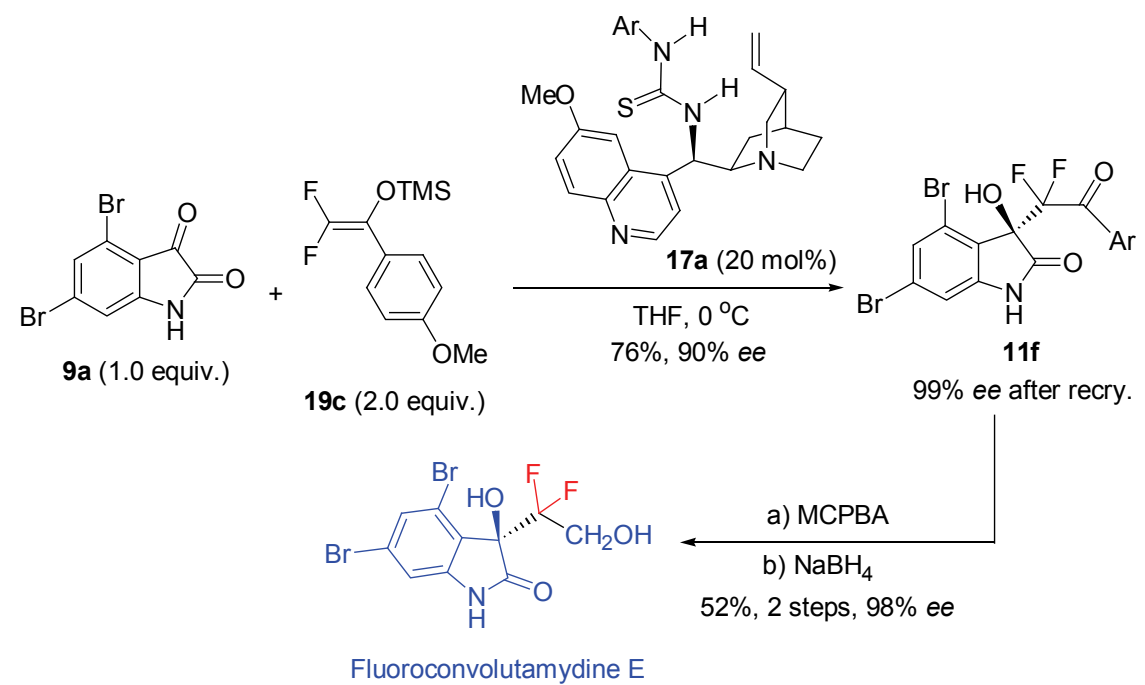

图 16 Fluoroconvolutamydine $\mathrm{E}$ 的全合成

Figure 16 The total synthesis of fluoroconvolutamydine E 
对比上述反应不难看出, 在实现醛或酮等羰基化合 物与靛红的直接 aldol 反应中, 有机小分子催化的方式 不论从反应的普适性、经济性和选择性方面均更为优越. 利用烯胺催化或布朗斯特碱对亲核的醛或酮的活化, 再 结合氢键给体对静红的活化, 可以很好地实现简单的醛 或酮与靛红的不对称直接 aldol 反应, 合成相关的天然 产物或关键中间体. 同时, 在关键反应步骤没有金属的 参与, 不用担心金属离子对产物的沾染. 事实上, 手性 金属催化实现的从简单的醛或酮出发的不对称 aldol 反 应的成功例子很少. 从 3-差基氧化吲哚手性砌块 $\mathbf{I}$ 的不 对称催化合成的反应实例, 可以管窥烯胺催化的发展, 有力地推动了 aldol 反应这一重要的 $\mathrm{C}-\mathrm{C}$ 键形成反应的 发展, 为 $\beta$-羟基酮或醛的不对称催化合成, 提供了经济 而高效的方法. 当然, 不对称烯胺催化还存在着诸如底 物范围不够广、催化剂用量较大等需要今后改进的方向.

\section{3-乙烯基取代的 3-羟基氧化吲哚手性砌块 II 的构建}

3-乙烯基取代的 3-羊基氧化吲哚手性砌块 II 同样也 非常重要, 既在 Convolutamydine D 等天然产物中存在, 同时 C(3)位的乙烯基还可以方便地进一步衍生化, 合成 一些重要的并环骨架. 这一手性砌块目前可通过金属催 化的烯基硅试剂对靛红的不对称加成反应和有机催化 的不对称 Morita-Baylis-Hillmann (MBH)反应来构建(图 17).

2009 年, Shibasaki 小组 ${ }^{[19]}$ 报道了首例催乙烯基三甲 氧基硅烷对静红的不对称加成反应, 在手性催化剂 23/CuF 的作用下, 3-乙烯基-3-羊基氧化吲哚化合物 $\mathbf{2 4}$ 可 以以高达 $99 \%$ 的产率和 $90 \%$ 的对映选择性得到. 他们发 现在反应体系中加入催化量的 $\mathrm{ZnF}_{2}$ 可以大大加快反应 速度. 作者认为该反应是烯基硅试剂通过转金属化反 应，生成相应的烯基铜活性物种作为真正的亲核试剂对 静红进行亲核加成. 这也是目前唯一的一例利用金属催 化来构建手性砌块 II 的例子(图 18).
MBH 反应是在手性中心方便引入乙烯基的重要反 应. 在过去十几年中, 特别是 Hatakeyama 等 ${ }^{[20]}$ 发现 $\beta$-isocupreidine $(\beta$-ICD, 25)这一高效催化剂以来, 基于 醛或醛亚胺的不对称 MBH 反应得到了广泛的研究, 但 利用酮作为亲电试剂的不对称 $\mathrm{MBH}$ 反应没有报道 ${ }^{[21]}$. 2010 年, 几个研究小组 ${ }^{[22]}$ 几乎同时报道了靛红作为亲 电试剂的不对称 $\mathrm{MBH}$ 反应来高选择性合成叔醇 II.

我们小组 ${ }^{[22 a, 20 b]}$ 首次实现了丙烯醛作为亲核试剂的 不对称 $\mathrm{MBH}$ 反应. 在 $\beta$-ICD 的作用下, 丙烯醛可与具有 不同取代基的靛红顺利反应，以中等到优秀的产率和对 映选择性得到产物 27. 使用丙烯醛作为亲核试剂的一个 重要优点是, 醛羰基可以进行多种衍生化, 如产物 27a 可通过选择性的还原分别得到内酯化合物 28 或 $3 a$-羊基 吲哚啉化合物 29 (图 19). 在这项工作基础上, 我们最近 还首次实现了丙烯醛对醛的不对称 MBH 反应，尽管只 适用于具有强吸电子取代基的芳香醛 ${ }^{[22 \mathrm{~g}]}$.

而施敏 ${ }^{[22 c]}$ 、卢一新 ${ }^{[22 \mathrm{~d}]}$ 和伍新燕等 ${ }^{[22 e, 22 f]}$ 分别独立地 实现了丙烯酸酯对静红的不对称 MBH 反应. 施敏小组 和陆易欣小组也是采用 $\beta-\mathrm{ICD}$ 催化剂, 分别实现了丙烯 酸 2-荎酯及丙烯酸芐酯对靛红的反应. 而伍新燕小组则 采用双功能的叔膦平面酰胺双功能催化剂 30 实现了活 性相对较低的丙烯酸甲酯、乙酯及正丁酯等与靛红的高 对映选择性 MBH 反应. 值得一提的是使用手性叔胺催 化剂 $\beta-\mathrm{ICD}$, 这些低活性的缺电子烯烃与责定红的反应较 慢, 而手性叔膦四方酸双功能催化剂 30 则很好地解决 了这个问题(图 20).

在合成 3-乙烯基取代的 3-差基氧化吲哚砌块中，金 属催化和有机催化各擅胜场, 显示了不同催化模式的特 点和使用范围. 通过对乙烯基的转金属化反应生成手性 的活性金属物种, 无疑需要金属催化剂的参与. 这也是 手性金属催化的传统应用范围. 但值得注意的是, 利用 手性 Lewis 碱活化硅试剂来实现不对称催化的反应，也 是有机催化的一个热点 ${ }^{[23]}$, 尽管有机催化的乙烯基硅试 剂对羰基化合物的加成还没有实现.

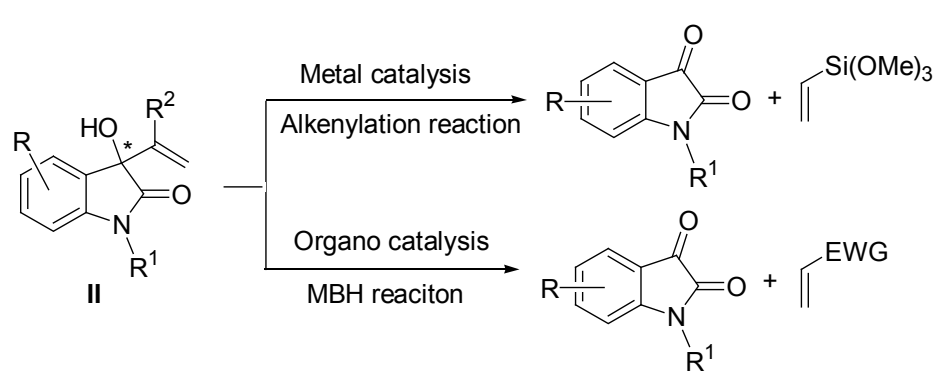

图 17 3-羟基氧化吲哚 II 的合成策略

Figure 17 Strategies for the synthesis of 3-hydroxyoxindole II 


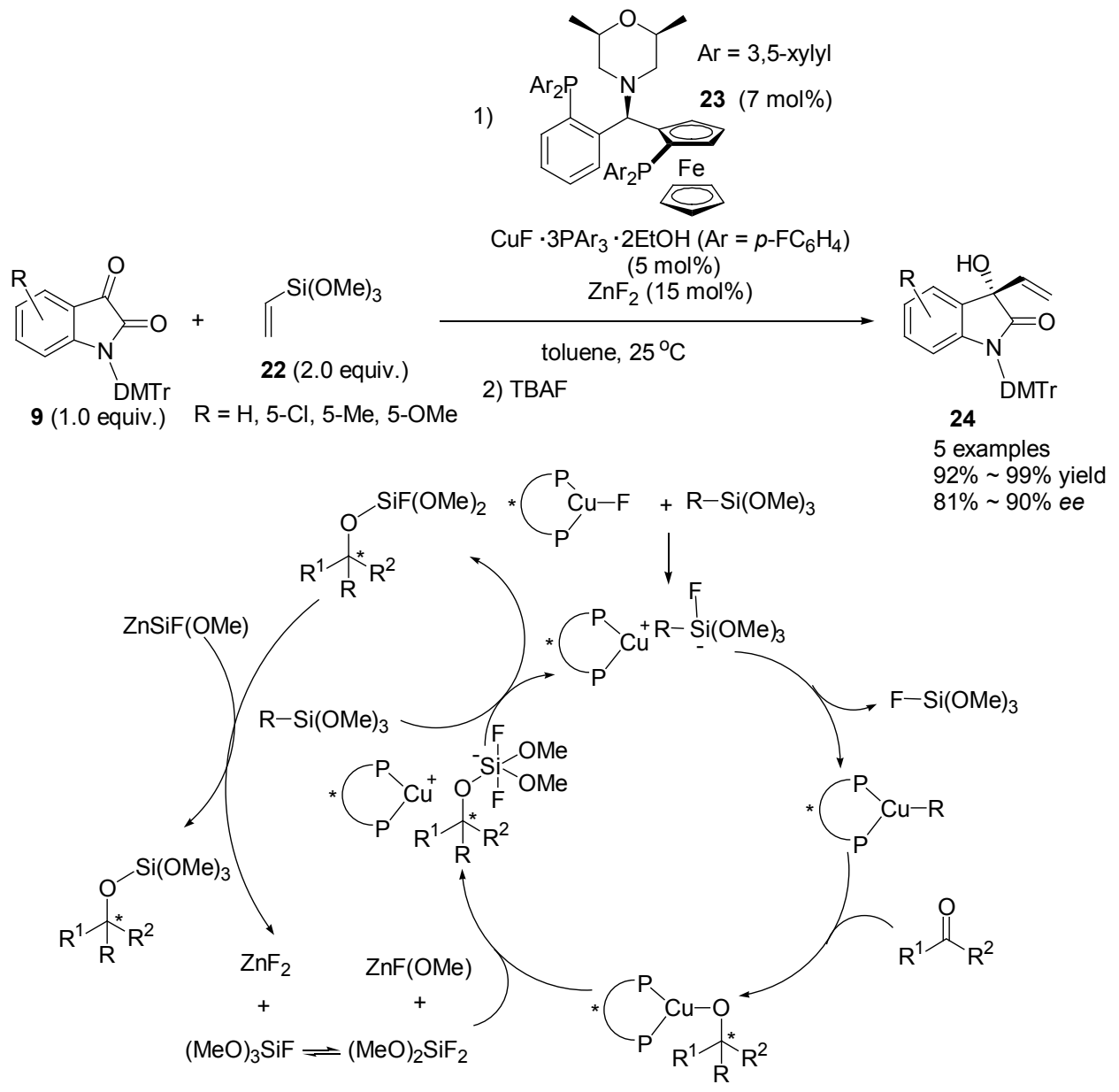

图 18 三甲氧基烯基硅 22 与靛红的加成反应

Figure 18 The addition of trimethoxyvinylsilane $\mathbf{2 2}$ with isatins<smiles>[R]c1ccc2c(c1)C(=O)C(=O)N2[R]</smiles>

9 (1.0 equiv.)

$\mathrm{R}=\mathrm{Me}, \mathrm{OMe}$, halogen; $\mathrm{R}^{1}=\mathrm{H}, \mathrm{Me}, \mathrm{Bn}$;

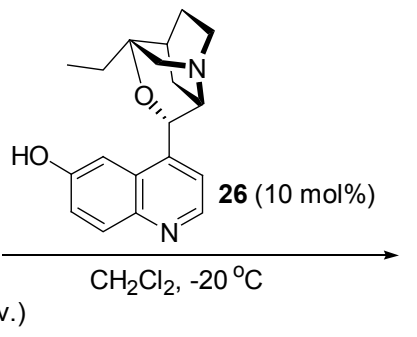

23 examples, $65 \% \sim 97 \%, 90 \% \sim 99 \%$ ee

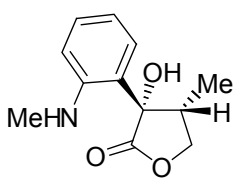

28: $81 \%, 98 \%$ ee<smiles>C=C[C@]1(O)C(=O)N(C)c2ccccc2[C@]1(O)C=O</smiles>

27a: $98 \%$ ee
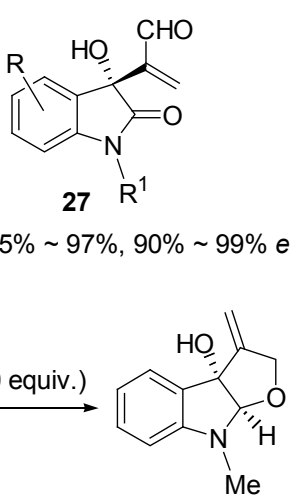

29: $75 \%, 98 \%$ ee

图 19 丙烯醛与靛红的 $\mathrm{MBH}$ 反应

Figure 19 MBH reaction of acrolein to isatins

另一方面, 不对称 MBH 反应则是有机催化的方法 展示其独特作用的舞台. 利用叔膦或者叔胺催化剂作为 Lewis 碱活化亲核试剂, $\alpha, \beta$-不饱和羰基化合物, 再结合
氢键给体对亲电试剂的活化，目前在开发不对称催化的 $\mathrm{MBH}$ 反应中得到广泛的应用. 单独使用手性金属催化 剂实现的不对称 $\mathrm{MBH}$ 反应还未见报道 ${ }^{[21]}$, 往往需要使 


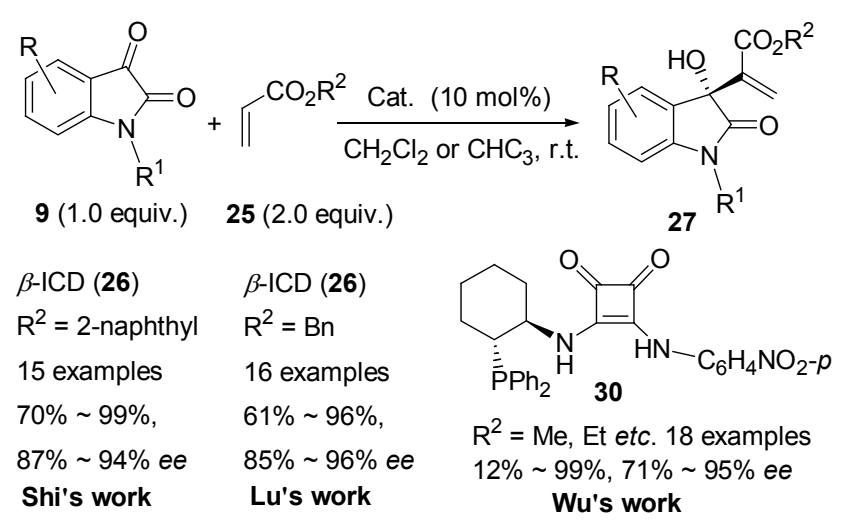

图 20 丙烯酸酯与静红的 $\mathrm{MBH}$ 反应

Figure 20 MBH reaction of acrylates to isatins

用路易斯碱对亲核试剂的活化，来协助手性金属催化剂 对亲电试剂的控制, 反应才能进行. 值得注意的是, 构 建 3-乙烯基取代的 3-羞基氧化吲哚砌块 II 的方法还不 多, 底物普适性还不够广, 还需要进行深入的研究.

\section{3-烯丙基取代的 3-羟基氧化吲哚手性砌块 III 的构建}

3-烯丙基取代的 3-羊基氧化吲哚手性砌块 III 可用 于如图 1 所示的 3,4 等多个天然产物的全合成. 目前, 这一手性砌块可通过以下二种策略进行不对称催化合 成: (1)靛红的不对称烯丙基化反应，(2) 3-烯丙基取代氧 化吲哚的 C(3)位氧化反应(图 21).

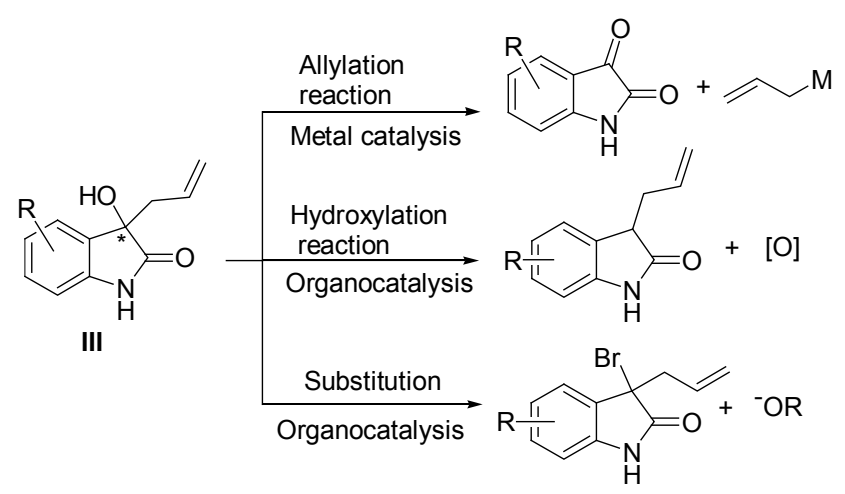

图 213 -羟基氧化吲哚 III 的合成策略

Figure 21 Strategies for the synthesis of 3-hydroxyoxindoles III

\section{1 烯丙基试剂对靛红的不对称亲核加成反应}

烯丙基金属试剂对靛红的不对称亲核加成反应是 构建 3-烯丙基取代的 3-羟基氧化吲哚的最直接的方法. 2006 年, Takayama 小组 ${ }^{[24]}$ 在分离到 CPC-1 后, 为确定天 然产物的绝对构型, 尝试了在 $(R)-\mathrm{Binol}$ 与 $\mathrm{Ti}(\mathrm{O} i-\mathrm{Pr})_{4}$ 形 成的手性催化剂作用下，四烯丙基锡与靛红的加成反 应, 并以 $42 \%$ 的对映选择性得到产物 32a. 经过二次重
结晶后, 产物 32a 的 ee 值可以高达 99\%, 并用于 CPC-1 的全合成. 这也是首次报道的烯丙基金属试剂对靛红的 烯丙基化反应(图 22).

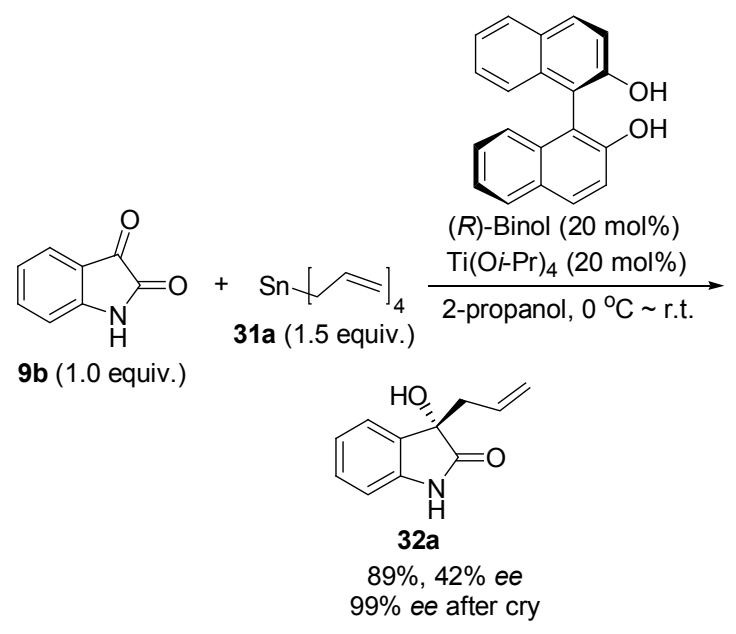

图 22 四烯丙基锡 31a 与靛红的烯丙化反应

Figure 22 The allylation reaction of tetraallylstannane 31a and isatins

由于四烯丙基锡毒性较大, 并需要当量使用. 为了 避免使用当量的烯丙基金属试剂, 2009 年, Krische 等 ${ }^{[25]}$ 尝试从烯丙基醋酸酯出发, 利用催化量的手性金属 Ir 催 化剂现场产生手性烯丙基金属试剂与靛红反应，高选择 性地实现了对靛红的烯丙基化反应. 7 种带有不同取代 基的 $N$-茮基保护的靛红均可高产率地转化为相应的目 标产物 32, 并且选择性均大于 $90 \%$ (图 23).

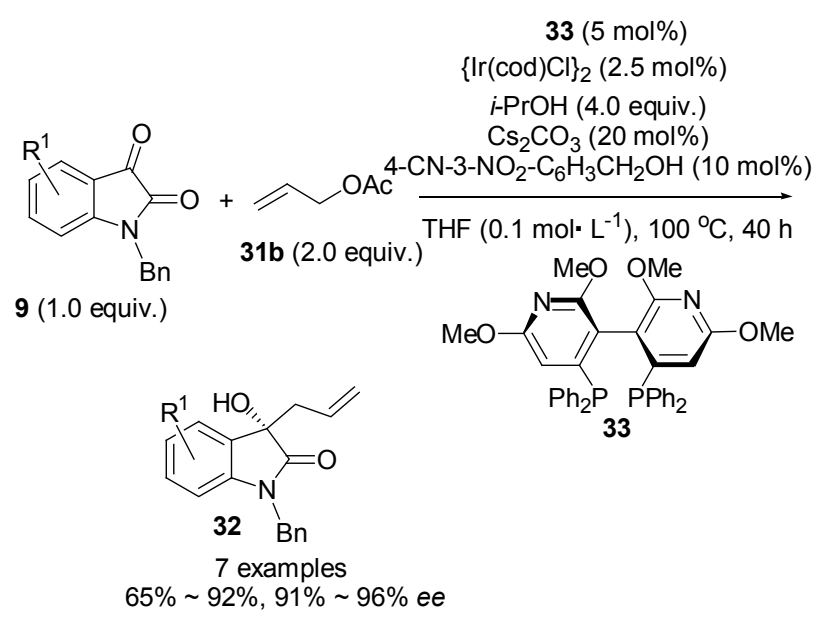

图 $23 \mathrm{Ir} / 33$ 催化的靛红与 $\mathbf{3 1 b}$ 的烯丙基化反应 Figure $23 \mathrm{Ir} / \mathbf{3 3}$ catalyzed the allylation reaction of isatins and 31b

2009 年，周其林等 ${ }^{[26 b]}$ 则希望从更简单的烯丙基化 试剂出发 ${ }^{[26 a]}$, 利用烯丙醇与嗍试剂现场生成相应的烯 丙基硼试剂, 再在螺环磷酰胺 34 与 $\mathrm{Pd}(\mathrm{OAc})_{2}$ 衍生的手 
性 Pd 催化剂作用下，与靛红反应生成相应的目标产物. 尽管反应的选择性中等，该方法为发展新的烯丙基化反 应提供了一种新的策略. 最近, 他们把这种极性反转的 策略应用到了对醛的高选择性烯丙基化反应中. 该方法 适用于氮上具有保护基的靛红的烯丙基化反应，如果使 用氮上没有保护基的底物, 则得到氮上和 $\mathrm{C}(3)$ 位羰基上 都发生烯丙基化的产物(图 24).

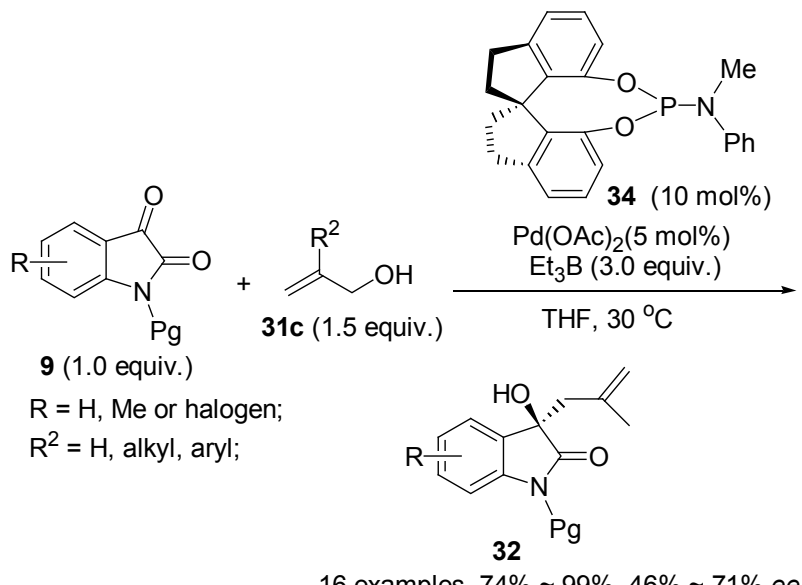

图 $24 \mathrm{Pd} / \mathbf{3 4}$ 催化的静红与 $\mathbf{3 1} \mathrm{c}$ 的烯丙基化反应

Figure $24 \mathrm{Pd} / \mathbf{3 4}$ catalyzed allylation reaction of isatins and 31c

Sakurai-Hosomi 反应，即烯丙基硅试剂对羰基化合 物的加成反应，是合成高烯丙醇的重要方法，因为硅试 剂毒性较小, 而且操作较方便. 该方法通常需使用手性 酸活化亲电试剂来促进反应，或者使用手性 Lewis 碱活 化烯丙基三氯硅烷与亲电试剂反应. 我们提出了一种利
用金属盐直接活化三甲基烯丙基硅烷这一最价廉的烯 丙基硅试剂，生成相应的烯丙基金属物种与羰基化合物 反应 ${ }^{[27]}$. 这是促进这一反应的一种新催化机制. 研究发 现 $\mathrm{Hg}\left(\mathrm{ClO}_{4}\right)_{2} \bullet \mathrm{H}_{2} \mathrm{O}$ 可以高效的活化烯丙基三甲基硅烷, 进而实现对靛红的加成反应. 这种活化方式很高效，只 需要 $1.0 \mathrm{~mol} \%$ 的手性 Binap 配体形成的录催化剂, 就可 高效催化反应，但产物的 $e e$ 值仅为中等(图 25).

Franz 等 ${ }^{[28]}$ 利用 $10 \mathrm{~mol} \%$ 的手性催化剂 33/Sc(OTf) 在 3 equiv. 的 TMSCl 和 $30 \mathrm{~mol}^{\circ}$ 的 $\mathrm{NaSbF}_{6}$ 的协助下，高 选择性地实现了 31d 与靛红的反应(图 25). 这一催化体 系还可应用于其他不同取代的烯丙基硅烷. 特别是高活 性的三甲基(2-甲基烯丙基)硅烷参与的反应，手性 Sc 催 化剂的用量可以降低到 $0.05 \mathrm{~mol} \%$. 尽管 Sc 催化剂在该 反应中取得了优秀的对映选择性, 但与录催化剂比较起 来, 不但催化剂用量高, 反应体系也远为复杂，需加入 $\mathrm{TMSCl}, \mathrm{NaSbF}_{6}$ 和 $4 \AA \mathrm{MS}$ 来协助反应进行, 这也说明手 性录催化值得进一步研究 ${ }^{[5 i]}$.

富电子的烯醇醚 19 与靛红的 ene 反应也可用于构 建手性砌块 III. 2011 年，冯小明等 ${ }^{[29]}$ 利用其小组发展的 优秀手性氮氧配体 37 与 $\mathrm{Mg}(\mathrm{OTf})_{2}$ 形成的催化剂, 高选 择性地实现了这一反应. 具有不同取代基的靛红均反应 良好，以中等到优秀的产率和优秀的对映选择性得到相 应的 3-烯丙基取代的 3-差基氧化吲哚 32. 所得到的产物 $32 \mathrm{a}$ 用四氢锂铝还原, 即可得到环戊酮并吲哚环化合物 38, 这一骨架在生物活性分子中广泛存在; 而产物 $32 \mathrm{~b}$ 通过简单的水解就可以 $98 \%$ 的产率, $97 \%$ 的 $e e$ 值得到天 然产物 $(R)$-convolutamydine $\mathrm{A}$ (图 26).

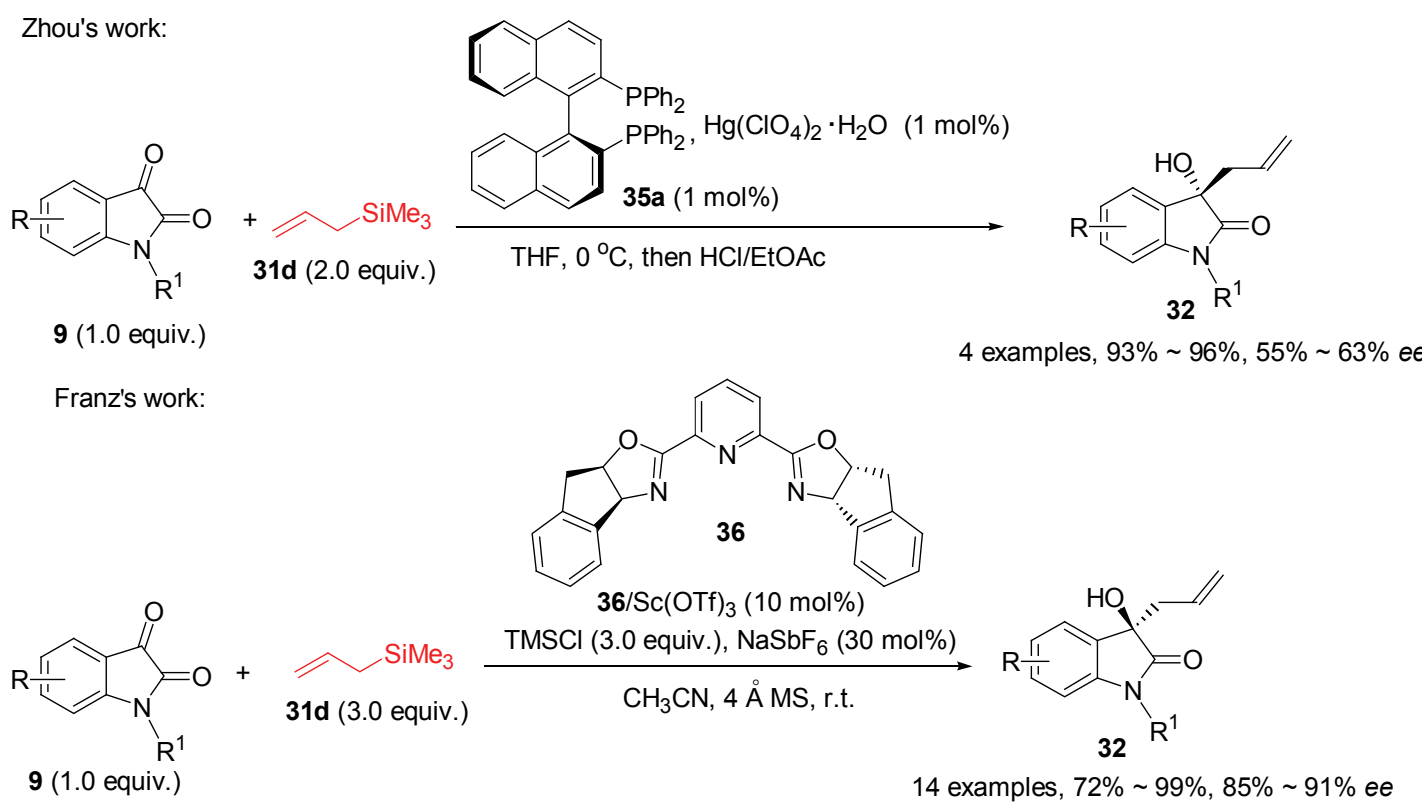

图 25 靛红的 Sakurai-Hosomi 反应

Figure 25 The Sakurai-Hosomi reaction of isatins 


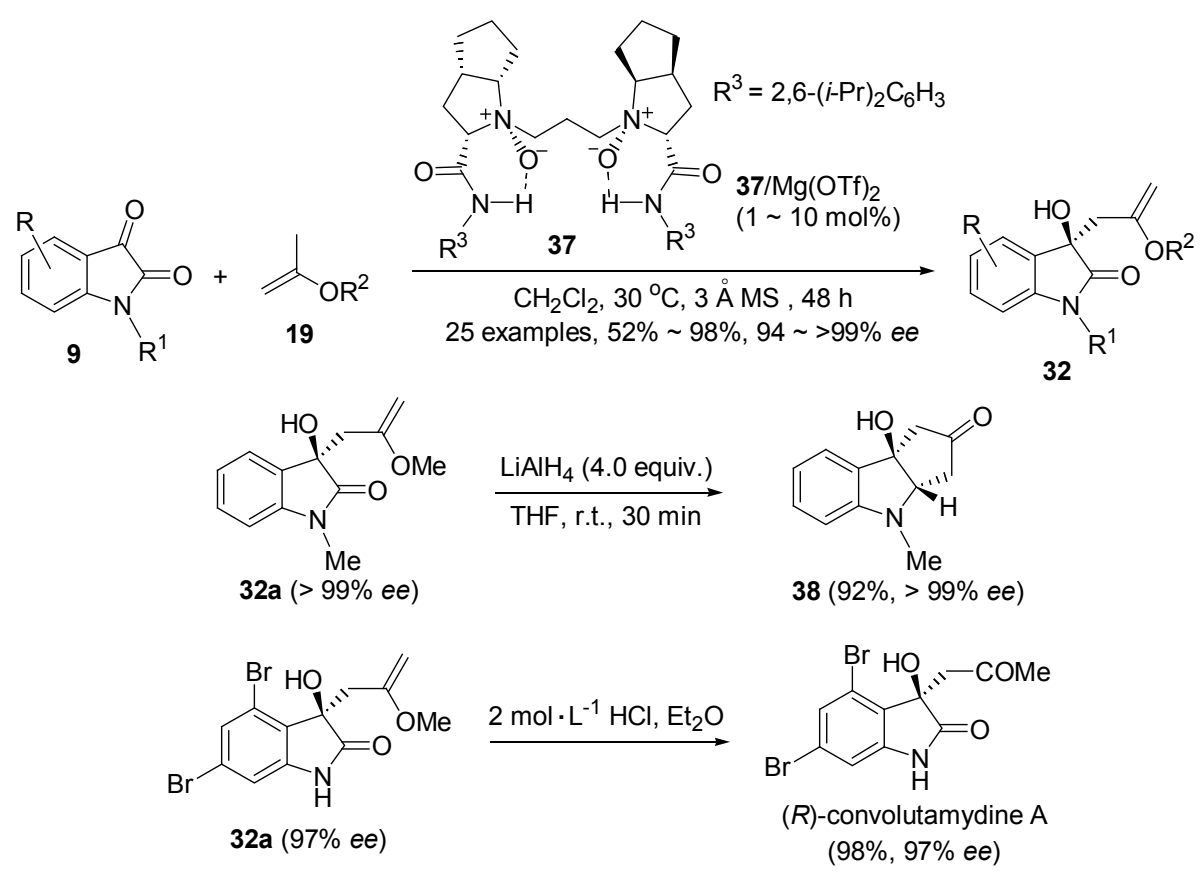

图 26 烯醇醚 19 与靛红的 carbonyl-ene 反应

Figure 26 The carbonyl-ene reaction of enol ether $\mathbf{1 9}$ and isatins

\subsection{3 -烯丙基取代的氧化吲哚的不对称羟基化反应}

有机催化的 3-取代的氧化吲哚的氧化反应也开发 出来制备 3-羟基季碳氧化吲哚. 在手性相转移催化下, 碱对 3-烯丙基取代的氧化吲哚挸氢活化后产生相应的 烯醇负离子与氧气反应, 即可形成手性 3-烯丙基取代的

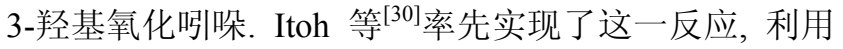
金鸡纳碱衍生的相转移催化剂 $\mathbf{4 0}$, 最高可取得 $85 \%$ 的对 映选择性. 作者还发现添加如 $(\mathrm{EtO})_{3} \mathrm{P}$ 类还原剂, 不但可 以加快反应速度, 还可以提高反应的对映选择性. 此外, Tan 小组 ${ }^{[31]}$ 也利用自己小组发展的相转移催化剂研究了 这一反应，最高取得 93\%的选择性(图 27).
除了使用氧气作为氧化剂外, Barbas III 小组 ${ }^{[322]}$ 还 利用奎尼丁衍生的双聚催化剂 43 实现了 3-烯丙基取代 的氧化吅哚与亚硝基苯 $\mathbf{4 0}$ 的氧化反应，反应的选择性 中等到优秀. 作者通过对照实验发现催化剂 43 中 $\mathrm{C}(9)$ 位的羟基与亚硝基苯中氮的氢键作用可能是反应的高 对映选择性及选择性对氧加成的关键(图 28).

2012 年, 袁伟成等 ${ }^{[32 b]}$ 则利用催化剂 47 实现了羟胺 与 3-溴取代氧化吲哚 $\mathbf{4 5}$ 的亲核取代反应, 所报道的一 个例子 $e e$ 值高达 $88 \%$. 产物 $\mathbf{4 8}$ 经过臭氧化及钯碳裂解 氮氧键后可以得到 1,3-二醇氧化吲哚 16a, 它是合成天 然产物如 CPC-1 及 donaxaridine 等关键中间体(图 29).

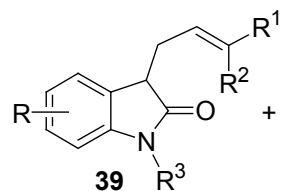

$39 \mathrm{R}^{3}$

Itoh's work:

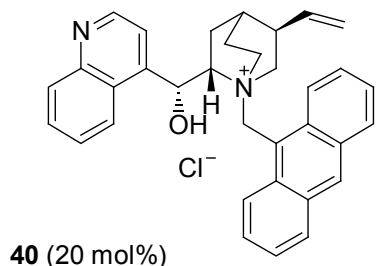

$40(20 \mathrm{~mol} \%)$

(EtO) $)_{3} \mathrm{P}$ ( 1.2 equiv.), $-20{ }^{\circ} \mathrm{C}, 2.5 \mathrm{~h}$

2 examples, up to $98 \%$ yield, $85 \%$ ee
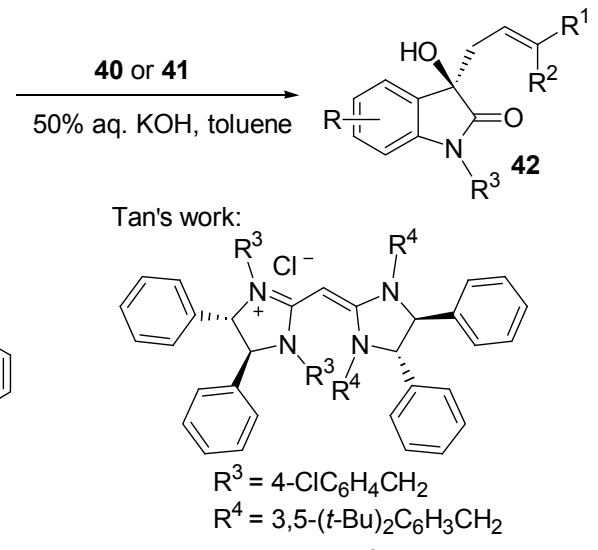

$41(5 \mathrm{~mol} \%),-60{ }^{\circ} \mathrm{C}, 72 \sim 96 \mathrm{~h}$

2 examples, up to $89 \%$ yield, $93 \%$ ee

图 27 3-烯丙基取代的氧化吲哚的氧化反应

Figure 27 The oxidation of 3-allyl substituted oxindoles 


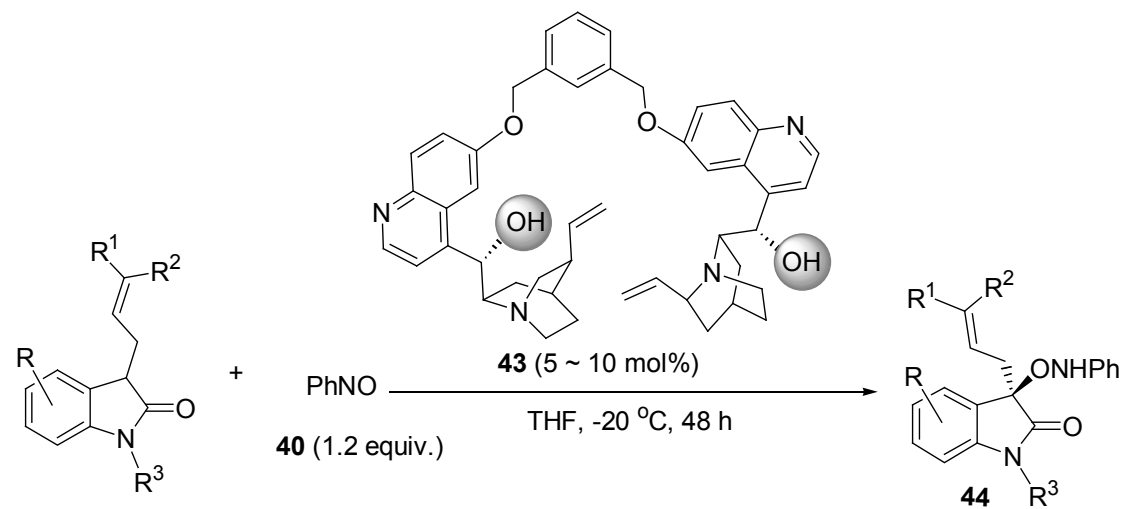

39 (1.0 equiv.)

5 examples, $65 \% \sim 86 \%, 73 \% \sim 92 \%$ ee

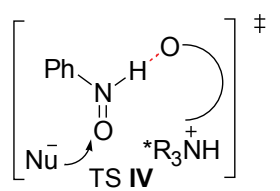

图 283 -烯丙基取代的氧化吲哚的胺氧化反应

Figure 28 Aminooxygenation of 3-allyl substituted oxindoles

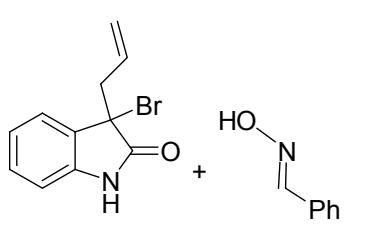

45 ( 1.0 equiv.) 46 (1.2 equiv.)

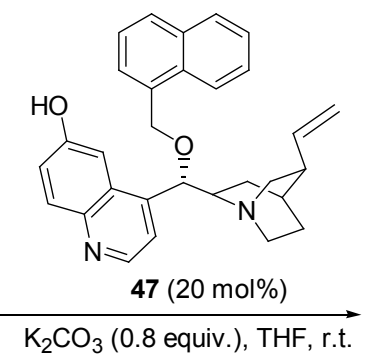

图 293 -溴取代氧化吲哚的羟基化反应

Figure 29 The hydroxylation of 3-bromineoxindole
在已有的构建 3-烯丙基取代的 3-羟基氧化吲哚手 性砌块 III 的不对称催化的合成方法中, 金属催化的方 法无疑更具有优势, 能够实现从基础原料静红出发, 利 用各种简单的烯丙基化试剂来实现高选择性合成, 不仅 选择性较高, 而且金属催化剂的用量可以降到 1.0 mol\%, 甚至更低. 有机催化的 3-烯丙基取代的氧化吲哚 的 C3 位氧化反应, 也是一种有用的方法, 甚至还可以 实现利用氧气这种清洁的氧化剂来开发反应. 但是这一 方法需要使用预先制备的 3-烯丙基取代的氧化吅哚, 不 但需要先制备不同取代基的氧化吲哚外, 还要再经过一 步烯丙基取代反应，而这一步反应受氧化吲哚的取代基 的影响较大, 产率通常不高 ${ }^{[33]}$.

\section{3-芳基取代的 3-羟基氧化吲哚手性砌块 IV 的 构建}

3-芳基取代的 3-着基氧化吲哚结构是一类具有重要 生物活性的化合物, 在药学研究中受到广发关注, 例如
口服非肽荷尔蒙生长激素药物 SM-130686 就含有这一 结构单元. 自从 Hayashi 等首次尝试该砌块的不对称催 化合成以来，目前已发展出三种不同的合成策略：(1)对 靛红的不对称芳基化反应; (2) 3-芳基取代的氧化吲哚直 接的氧化反应; (3)金属催化的 $\alpha$-酮酰胺的分子内环化反 应(图 30).

\section{1 靛红的不对称芳基化反应}

芳基硼酸作为良好的芳基化试剂在不对称合成中 得到了广泛的运用. 利用芳基嗍试剂对静红的加成是构 建 3-芳基 3-羟基氧化吲哚的一种直接方法. 到目前为止 已经有多个小组对此进行了研究 ${ }^{[34]}$. Hayashi 等 ${ }^{[34 a]}$ 于 2006 年, 利用手性催化剂 $(R)-50 / \mathrm{Rh}$, 在温和的条件下以 高达 98\%的产率和 $91 \%$ 的对映选择性首次实现了这一 芳基化反应(图 31).

此外，秦勇等 ${ }^{[346]}$ 利用 $\mathrm{Pd} / \mathbf{5 2}$ 络合物同样实现了芳基 硼酸对 $N$-芐基保护靛红的不对称加成反应，反应的对映 选择性最高达 73\% (图 32). 


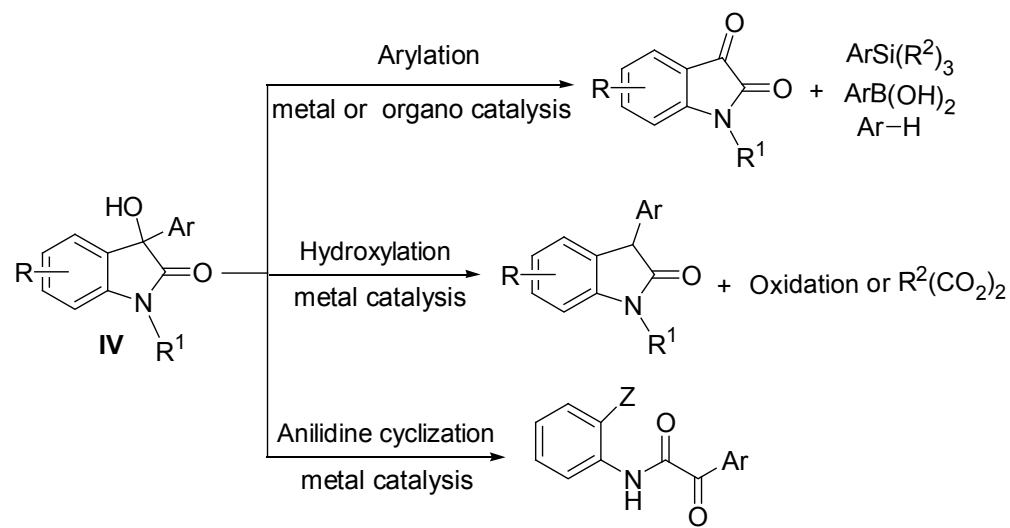

图 30 3-芳基取代的 3-羊基氧化吲哚的合成策略

Figure 30 Strategies for the synthesis of 3-aryl substituted 3-hydroxyloxidoles

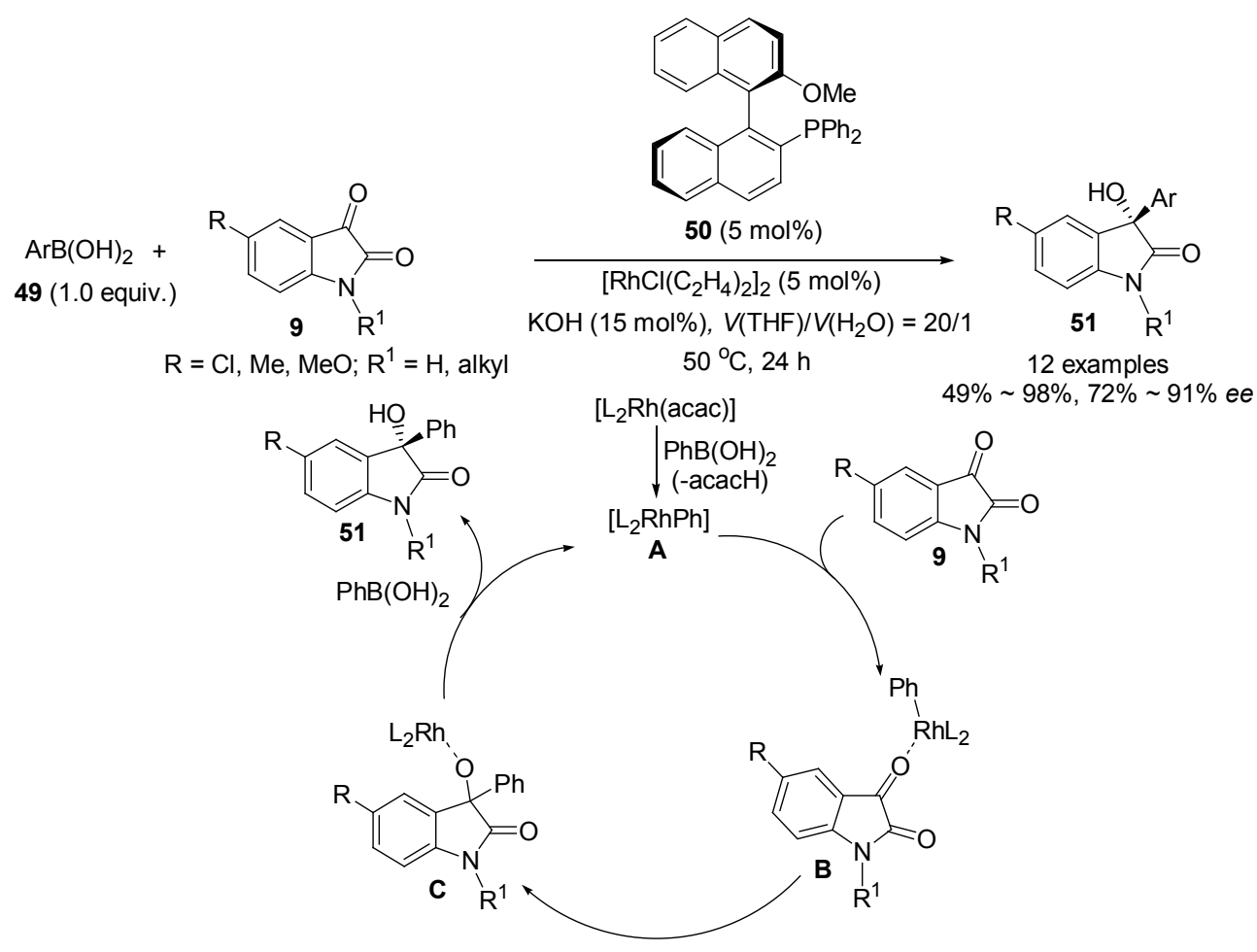

图 31 芳基嗍酸对靛红的加成反应

Figure 31 The addition of arylbornic acids to isatins

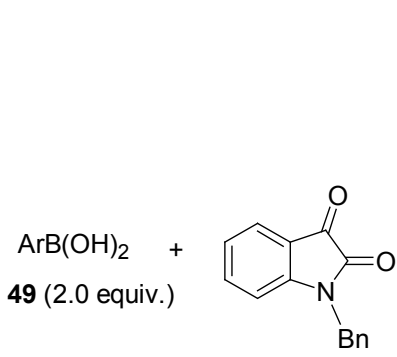

9c (1.0 equiv.)
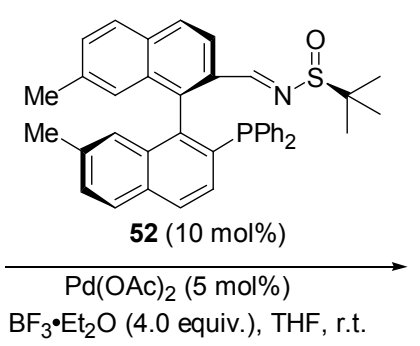

$\mathrm{BF}_{3} \cdot \mathrm{Et}_{2} \mathrm{O}$ (4.0 equiv.), THF, r.t.

11 examples, up to $78 \%$ yield, $73 \%$ ee

图 32 芳基硼酸对靛红的加成反应

Figure 32 The addition of arylbornic acids to isatins

芳基硅试剂对靛红的不对称加成反应，也得到了研 究. 2009 年, Shibasaki 等 ${ }^{[19]}$ 利用催化剂 23 实现了四例高 
选择性和产率的芳基硅试剂与靛红的不对称芳基化反 应(图 33). 如图 18 所示该方法还适用于烯基硅试剂对靛 红的反应.

尽管芳基硼化物对靛红的加成反应具有较好的底 物普适性，但对于对于某些富电子的(杂)芳香取代基, 则可通过更具原子经济性的富电子芳环与靛红的傅克 反应来制备. 2010 年, Franz 等 ${ }^{[35 a]}$ 利用噁唑啉配体衍生 的手性锌催化剂, 实现了吲哚、呋喃和芳香醚等富电子 芳香烃与靛红的不对称傅克反应，以 $73 \% \sim 99 \%$ 的产率 及 $80 \% \sim 99 \%$ 的 $e e$ 值得到目标产物 51. 吡咯作为亲核试 剂的反应难度较大, 需要克服区域选择性及自身聚合等 问题, 他们发展了手性催化剂 36/In $(\mathrm{OTf})_{3}$, 实现了吡咯 $\mathrm{C} 2$ 位的选择性傅克反应 ${ }^{[35 b]}$ (图 34).

随后, 王卫小组 ${ }^{[36]}$ 实现了有机催化的吲哚与靛红的 Friedel-Crafts 反应. 在 cupreine 54 的催化下，具有不同 取代基的靛红及吲哚均能顺利进行反应，以 $68 \% \sim 97 \%$
的产率及 $76 \%$ ～91\%的对映选择性得到相应的产物 51 . 作者认为催化剂 cupreine 54 起到双重活化作用，6 位 $\mathrm{OH}$ 和 9 位 $\mathrm{OH}$ 通过氢键活化靛红, 而叔胺则与吲哚形 成氢键, 导向吲哚从靛红羰基的 $r e$ 面进攻, 从而得到 $(S)$ 构型的产物. 因此，该反应局限于 $N$ 上不带保护基的吲 哚(图 35). 最近, Chauhan 等 ${ }^{[37]}$ 也用相似的催化剂实现了 这一高对映选择性的 Fridel-Crafts 反应.

\subsection{3-芳基取代的氧化吲哚的不对称羟基化反应}

3-位芳基取代的氧化哚的不对称催化氧化反应也 是构一种有效的合成方法. 2006 年, Shibata 等 ${ }^{[38]}$ 采用 oxaziridine 56 作为氧化剂, $\mathrm{DBFOX} / \mathrm{Zn}(\mathrm{II})$ 作为催化 剂，以中等到优秀的产率和选择性实现了这一反应(图 36). 该方法还适用于 3-烷基取代的氧化吲哚. 而图 27 所示的有机催化的氧化方法只适用于 3-烷基取代的氧 化吲哚. 显然这一金属催化的方法具有更好的底物普适 性.

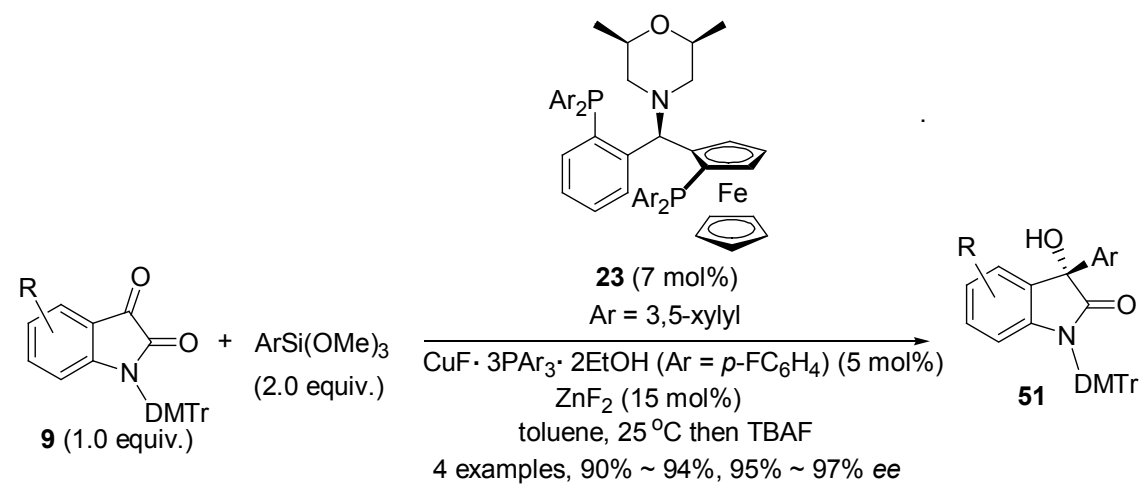

图 33 芳基三甲氧基硅烷与靛红的加成反应

Figure 33 The addition of aryltrimethoxysilanes to isatins

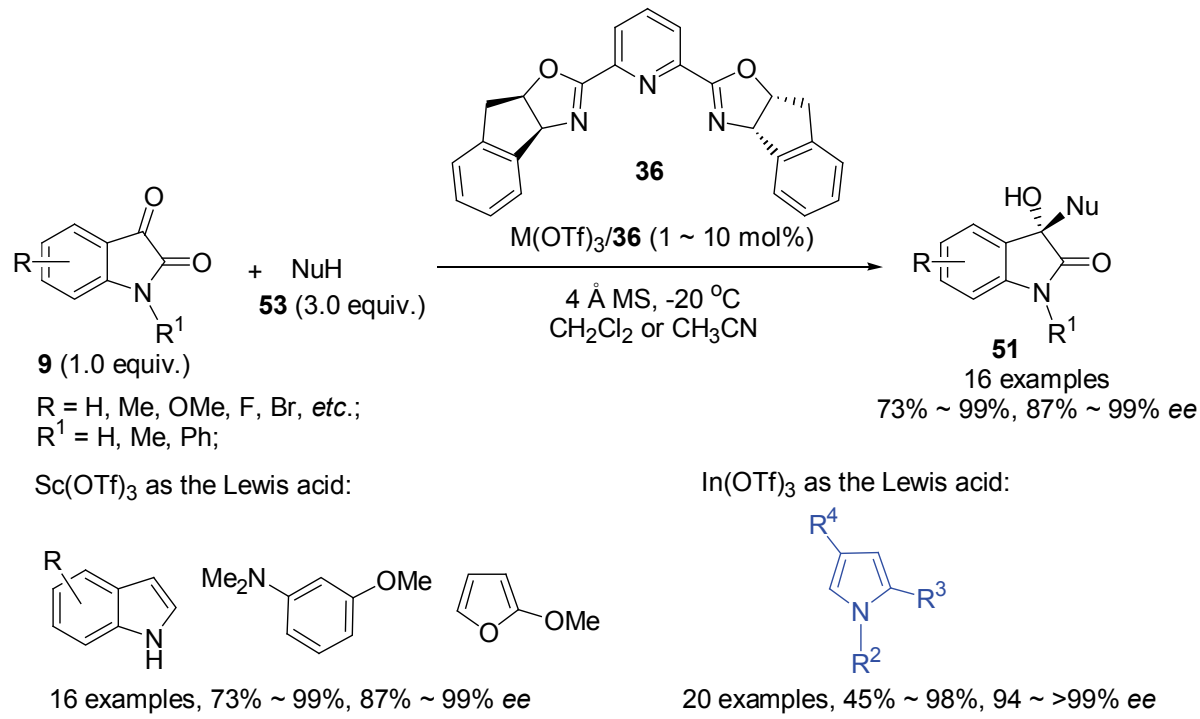

图 34 靛红与 53 的 Friedel-Crafts 反应

Figure 34 The Friedel-Crafts reaction of $\mathbf{5 3}$ and isatins 

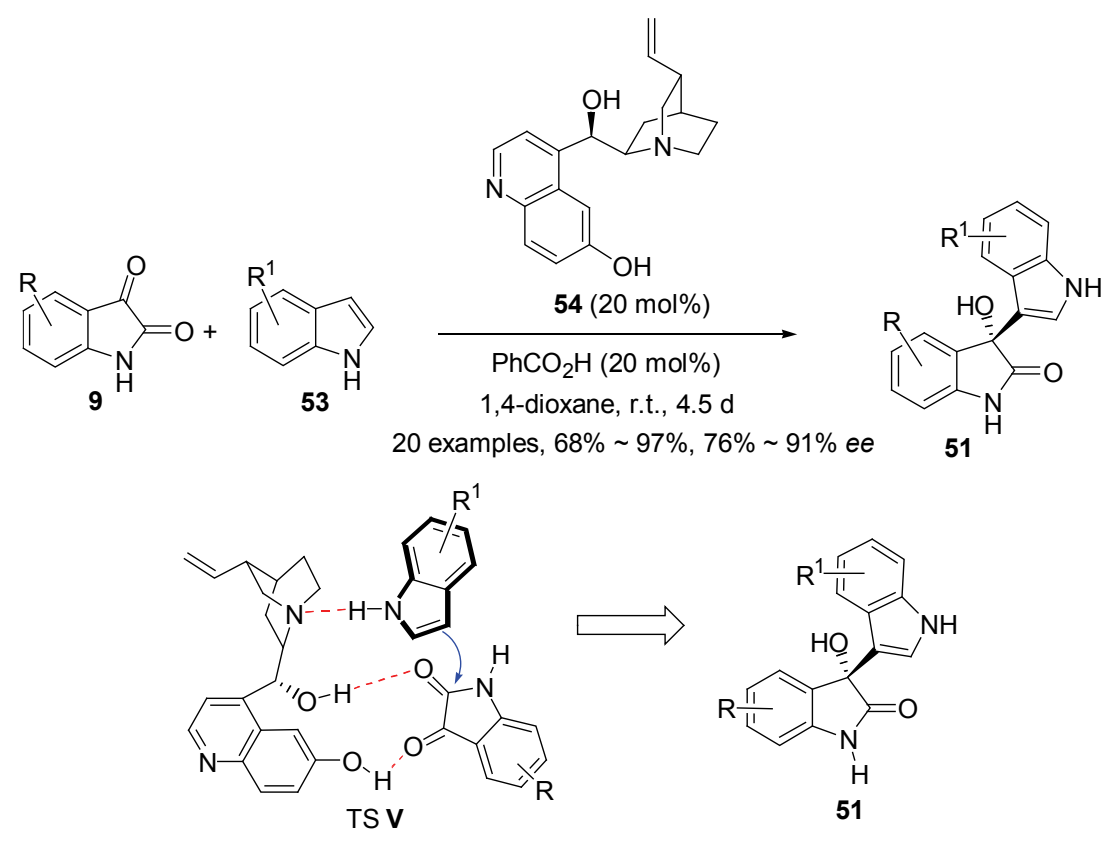

图 35 吲哚与靛红的 Fridel-Crafts 反应

Figure 35 The Fridel-Crafts reaction of indole and isatins

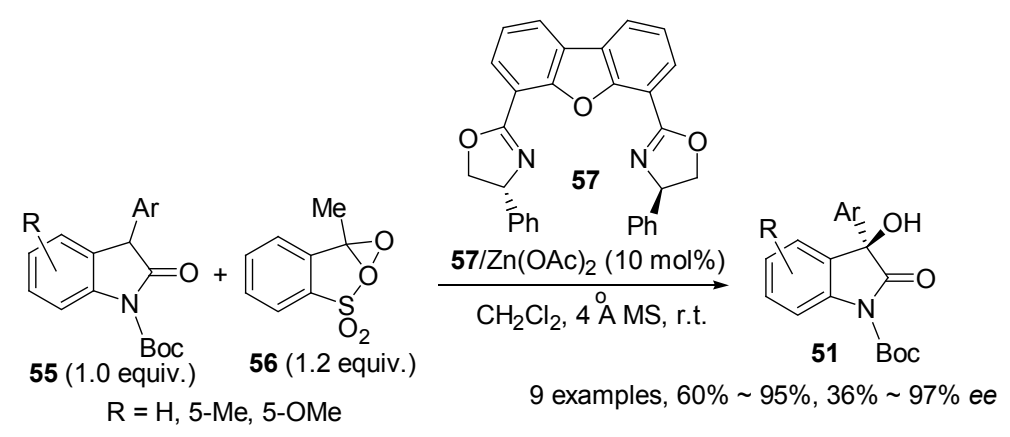

图 36 3-芳基取代氧化吲哚 55 的氧化反应

Figure 36 The oxidation of 3-aryl substituted oxindoles $\mathbf{5 5}$

另外, 3-芳基取代的氧化吲哚与过氧化合物的反应 也是构建 3-芳基取代的 3-羟基氧化吲哚的一种间接的 方法. 2011 年, Antilla 小组 ${ }^{[39]}$ 利用手性 VAPOL 钻的磷酸 盐 59 为催化剂, 实现了 3-芳基氧化吲哚与过氧苯甲酰 的苯甲酸化反应. 对于带不同取代基的底物, 反应均可 以优秀的对映选择性( $91 \sim>99 \% e e$ ) 得到相应的苯甲酸 化产物, 经过 DIBAL-H 的还原即可顺利的转化成 3-芳 基取代的 3-羊基取代氧化吲哚(图 37).

\section{$4.2 \alpha$-酩酰胺的分子内不对称环化反应}

手性金属催化的 $\alpha$-酮酰胺的不对称分子内环化反 应，也可用于构建 3-芳基取代的 3-羟基氧化吲哚. 2009 年, Shibasaki 小组 ${ }^{[19]}$ 在研究研究药物分子 SM-130686 的 不对称全合成时, 发现 C(4)位大位阻的三氟甲基的存 在，使得利用芳基硅试剂对静红的不对称亲核加成反应 构建 $\mathrm{C}(3)$ 位的季碳手性中心难以实现, 因此他们发展了
首个分子内的 $\alpha$-酩酰胺与硼酸酯的不对称环化反应，成 功的实现了药物分子 SM-130686 的全合成(图 38).

随后，该小组 ${ }^{[40]}$ 又报道了 Difluorophos (35b) 衍生的 手性钯催化剂实现的邻位三氟甲磺酸酯取代的 $\alpha$-酮酰 胺的不对称分子内环化反应，以高达 $92 \%$ 的产率和 $99 \%$ 的 $e e$ 值得到相应的 3-差基氧化吲哚化合物 51. 值得一 提的是这一方法可以用于同时构建 3-芳基或烷基取代 的 3-羟基氧化吲哚, 与他们组之前发展的方法相比, 底 物范围得到了扩展.

总体上来说，在手性砌块 IV 的不对称构建中，不对 称金属催化的方法得到更为广泛的研究和运用, 具有其 独特的优势, 无论是靛红直接的不对称芳基化反应、3芳基取代的氧化吲哚的氧化着基化及 $\alpha$-酮酰胺的分子 内环化反应，都可以很好的实现富电子及缺电子芳基取 代的 3-差基氧化弜哚化合物的构建. 而有机催化的方法 目前只有氮上无保护基的吲哚对靛红的加成这一例子, 


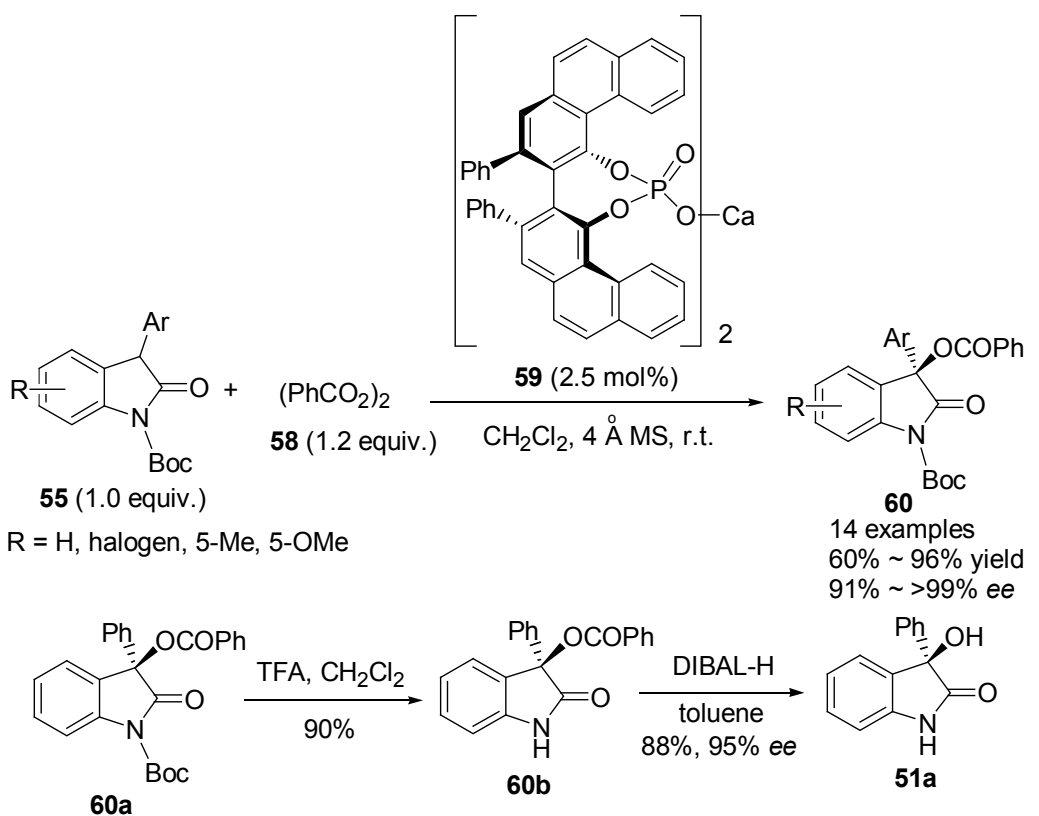

图 3755 对映选择性的苯甲酸化反应

Figure 37 Enantioselective benzoyloxylation of $\mathbf{5 5}$

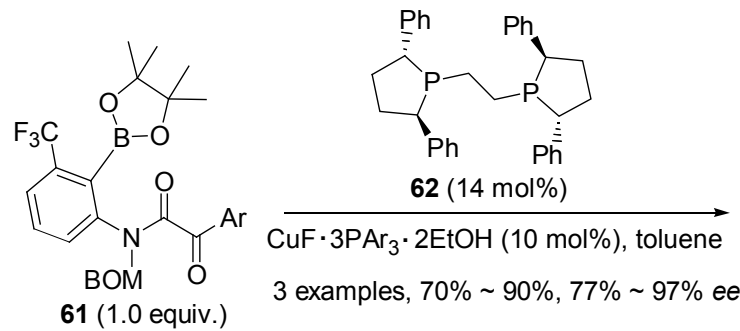

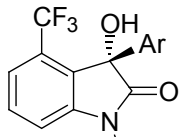

51 BOM

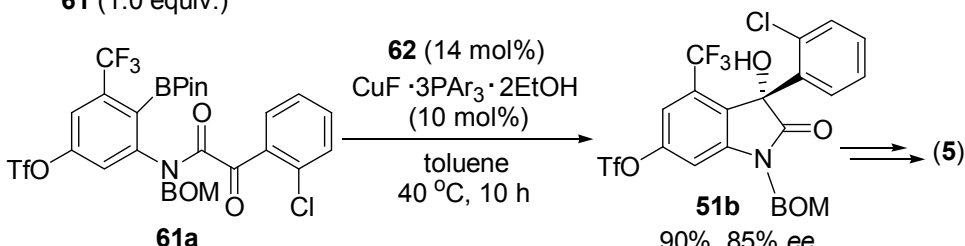

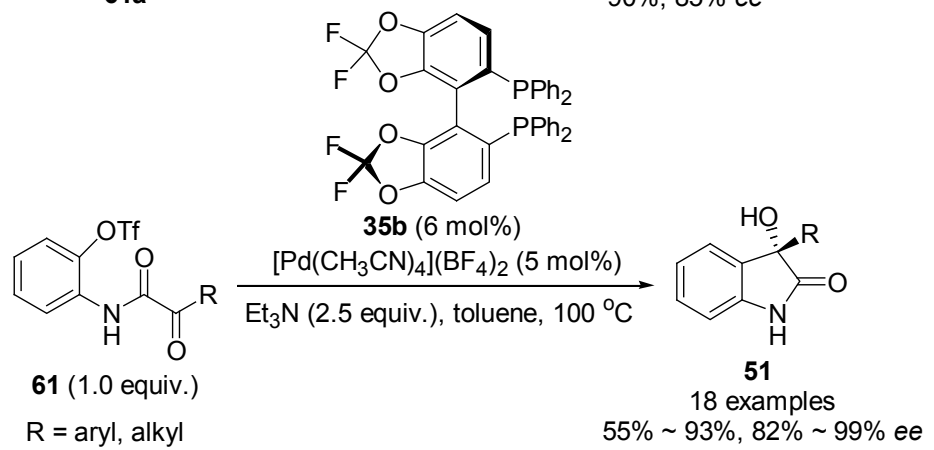

$90 \%, 85 \%$ ee

图 $38 \alpha$-酮酰胺的分子内不对称芳基化反应

Figure 38 Enantioselective intramolecular arylation of $\alpha$-ketoamides

底物范围不够广. 另外, 与不对称有机催化相比, 不对 称金属催化的方法的反应速度更快，以及催化剂用量较 小. 当然不对称金属催化的反应也存在发展空间，如发 展更为方便易得的手性配体来实现高选择性等.

\section{5 结论与展望}

综上所述，对映选择性地合成 3-取代的 3-羊基氧化 吲哚手性砌块自 2005 年来发展迅速. 不论是金属催化 
还是有机催化的方法都在这一领域得到了广泛的运用. 通过对 4 种典型的 3-羊基氧化吲哚手性砌块 I IV 的不 对称催化的合成方法的介绍, 不难看出有机催化的方法 在手性砌块 I, II 的合成中占有优势, 而金属催化的方法 则在手性砌块 III, IV 的合成中更具优势. 这是由这 2 种 不同催化模式本身的特点所决定的，同时也进一步说明 金属催化与有机催化的互补性. 这也启示不对称催化的 研究工作者在开发新的不对称反应，在设计开发某一类 特殊结构的手性化合物的时候, 要善于根据产物的结构 特点以及拟采用的反应底物的可能活化方式来选择采 用适当的手性催化方法.

尽管构建 3-取代的 3-羟基氧化吲哚的不对称催化 的合成方法在近年来得到快速发展, 但这一领域的研究 还有巨大的发展空间. 特别是目前, 新药研发迫切需要 基于生物活性分子的优势骨架的结构多样的化合物, 以 供活性篎选. 因此, 发展多样性和高选择性合成 3-取代 的 3-羟基氧化吲哚的方法, 仍然是一项具有重要应用价 值的研究工作. 无疑, 根据金属催化和有机小分子催化 的特点, 选择适当的起始原料, 来设计开发新的不对称 催化反应, 有助于实现高效、经济和快速的合成 3-取代 的 3-羟基氧化吲哚.

\section{References}

[1] For reviews, see: (a) Marti, C.; Carreira, E. M. Eur. J. Org. Chem. 2003, 2209 .

(b) Lin, H.; Danishefsky, S. J. Angew. Chem., Int. Ed. 2003, 42, 36. (c) Galliford, C. V.; Scheidt, K. A. Angew. Chem., Int. Ed. 2007, 46, 8748 .

(d) Steven, A.; Overman, L. E. Angew. Chem., Int. Ed. 2007, 46, 5488 .

(e) Trost, B. M.; Brennan, M. K. Synthesis 2009, 3003.

(f) Peddibhotla, S. Curr. Bioact. Compd. 2009, 5, 20.

For selected examples: (g) Liu, Y.; Zhang, L.; Jia, Y. Tetrahedron Lett. 2012, 53, 684.

(h) Kamano, Y.; Zhang, H.-P.; Ishihara, Y.; Kizu, H.; Komiyama, K.; Petti, G. R. Tetrahedron Lett. 1995, 36, 2783.

(i) Kamano, Y.; Kotake, A.; Hashima, H.; Hayakawa, I.; Hiraide, H.; Zhang, H.; Kizu, H.; Komiyama, K.; Hayashi, M.; Pettit, G. R. Collect. Czech. Chem. Commun. 1999, 64, 1147.

(j) Kitajima, M.; Mori, I.; Arai, K.; Kogure, N.; Takayama, H. Tetrahedron Lett. 2006, 47, 3199.

(k) Kohno, J.; Koguchi, Y.; Nishio, M.; Nakao, K.; Kuroda, M.; Shimizu, R.; Ohnuki, T.; Komatsubara, S. J. Org. Chem. 2000, 65, 990.

(1) Tokunaga, T.; Hume, W. E.; Umezome, T.; Okazaki, K.; Ueki, Y.; Kumagai, K.; Hourai, S.; Nagamine, J.; Seki, H.; Taiji, M.; Noguchi H.; Nagata, R. J. Med. Chem. 2001, 44, 4641.

[2] (a) Hewawasam, P.; Meanwell, N. A.; Gribkoff, V. K.; Dworetzky, S. I.; Boissard, C. G. Bioorg. Med. Chem. Lett. 1997, 7, 1255. (b) Hewawasam, P.; Erway, M.; Moon, S. L.; Knipe, J.; Weiner, H.; Boissard, C. G.; Post-Munson, D. J.; Gao, Q.; Huang, S.; Gribkoff, V. K.; Meanwell, N. A. J. Med. Chem. 2002, 45, 1487.

(c) Barroso, S.; Blay, G.; Cardona, L.; Fernandez, I.; Garcia B.; Pedro, J. R. J. Org. Chem. 2004, 69, 6821. (d) Nakamura, T.; Shirokawa, S.-I.; Hosokawa, S.; Nakazaki A.; Kobayashi, S. Org. Lett. 2006, 8, 677.

[3] (a) Zhou, F.; Liu, Y.-L.; Zhou, J. Adv. Synth. Catal. 2010, 352, 1381.

(b) Shen, K.; Liu, X.; Lin, L.; Feng, X. Chem. Sci. 2012, 3, 327.

(c) Dalpozzo, R.; Bartoli, G.; Bencivenni, G. Chem. Soc. Rev. 2012, $41,7247$.

(d) Ball-Jones, N. R.; Badillo, J. J.; Franz, A. K. Org. Biomol. Chem. 2012, 10, 5165 .

(e) Rios, R. Chem. Soc. Rev. 2012, 41, 1060.

(f) Singh, G. S.; Desta, Z. Y. Chem. Rev. 2012, 112, 6104.

(g) Kumar, A.; Chimni, S. S. RSC Adv. 2012, 2, 9748.

[4] (a) Shi, Y. Acc. Chem. Res. 2004, 37, 488.

(b) Vachal, P.; Jacobsen, E. N. Org. Lett. 2000, $2,867$.

(c) Vachal, P.; Jacobsen, E. N. J. Am. Chem. Soc. 2002, 124, 10012.

(d) List, B.; Lerner, R. A.; Barbas III, C. F. J. Am. Chem. Soc. 2000, 122, 2395 .

(e) Ahrendt, K. A.; Borths, C. J.; MacMillan, D. W. C. J. Am. Chem. Soc. 2000, 122, 4243.

[5] (a) Ding, M.; Zhou, F.; Liu, Y.-L.; Wang, C.-H.; Zhao, X.-L.; Zhou, J. Chem. Sci. 2011, 2, 2035.

(b) Qian, Z.-Q.; Zhou, F.; Du, T.-P.; Wang, B.-L.; Ding, M.; Zhao, X.-L.; Zhou, J. Chem. Commun. 2009, 6753.

(c) Zhou, F.; Cao, Z.-Y.; Zhang, J.; Yang, H.-B.; Zhou, J. Chem.-Asian J. 2012, 7, 233.

(d) Ding, M.; Zhou, F.; Qian, Z.-Q.; Zhou, J. Org. Biomol. Chem. 2010, 8, 2912.

(e) Zhou, F.; Ding, M.; Liu, Y.-L.; Wang, C.-H.; Ji, C.-B.; Zhang, Y.-Y.; Zhou, J. Adv. Synth. Catal. 2011, 353, 2945.

(f) Liu, Y.-L.; Zhou, J. Chem. Commun. 2013, 49, 2022.

(g) Yu, J.-S.; Zhou, F.; Liu, Y.-L.; Zhou, J. Beilstein J. Org. Chem. 2012, 8,1360

(h) Zhou, F.; Zeng, X.-P.; Zhao, X.-L.; Zhou, J. Chem. Commun 2013, 49, 2022.

(i) Cao, Z.-Y.; Zhou, F.; Yu, Y.-H.; Zhou, J. Org. Lett. 2013, 15 , 42.

(j) Zhou, F.; Ding, M.; Zhou, J. Org. Biomol. Chem. 2012, 10, 3178 .

(k) Liu, Y.-L.; Zhou, F.; Cao, J.-J.; Ji, C.-B.; Ding, M.; Zhou, J. Org. Biomol. Chem. 2010, 8, 3847.

(1) Zhu, F.; Zhou, F.; Cao, Z.-Y.; Wang, C.; Zhang, Y.-X.; Wang, C.-H.; Zhou, J. Synthesis 2012, 3129.

[6] For a review, see: Mukherjee, S.; Yang, J. W.; Hoffmann, S.; List, B. Chem. Rev. 2007, 107, 5471.

[7] (a) Luppi, G.; Cozzi, P. G.; Monari, M.; Kaptein, B.; Broxterman, Q. B.; Tomasini, C. J. Org. Chem. 2005, 70, 7418.

(b) Luppi, G.; Monari, M.; Corrěa, R. J.; Violante, F. de A.; Pinto, A. C.; Kaptein, B.; Broxterman, Q. B.; Garden, S. J.; Tomasini, C. Tetrahedron 2006, 62, 12017.

(c) Hernández, J. G.; García-López, V.; Juaristi, E. Tetrahedron 2012, 68, 92 .

(d) Malkov, A. V.; Kabeshov, M. A.; Bella, M.; Kysilka, O.; Malyshev, D. A.; Pluháčková, K.; Kočovský, P. Org. Lett. 2007, 9, 5473. (e) Shen, C.; Shen, F.; Xia, H.; Zhang, P.; Chen, X. Tetrahedron: Asymmetry 2011, 22, 708.

(f) Ricci, A.; Bernardi, L.; Gioia, C.; Vierucci, S.; Robitzer, M.; Quignard, F. Chem. Commun. 2010, 46, 6288.

(g) Chen, J.-R.; Liu, X.-P; Zhu, X.-Y.; Li, L.; Qiao, Y.-F.; Zhang, J.-M.; Xiao, W.-J. Tetrahedron 2007, 63, 10437.

(h) Chen, G.; Wang, Y.; He, H.; Gao, S.; Yang, X.; Hao, X. Heterocycles 2006, 68, 2327.

(i) Nakamura, S.; Hara, N.; Nakashima, H.; Kubo, K.; Shibata, N.; 
Toru, T. Chem. Eur. J. 2008, 14, 8079.

[8] (a) Raj, M.; Veerasamy, N.; Singh, V. K. Tetrahedron Lett. 2010, $51,2157$.

For the other examples see: (b) Liu, Y.; Gao, P.; Wang, J.; Sun, Q.; Ge, Z.; Li, R. Synlett 2012, 1031.

[9] Allu, S.; Molleti, N.; Panem, R.; Singh, V. K. Tetrahedron Lett. 2011, 52, 4080 .

[10] Hara, N.; Nakamura, S.; Shibata, N.; Toru, T. Chem. Eur. J. 2009, 15,6790 .

[11] (a) Xue, F.; Zhang, S.; Liu, L.; Duan, W.; Wang, W. Chem.-Asian J. 2009, 4, 1664.

For the other examples see: (b) Itoh, T.; Ishikawa, H.; Hayashi, Y. Org. Lett. 2009, 11, 3854.

(c) Chen, W.-B; Du, X.-L.; Cun, L.-F.; Zhang, X.-M.; Yuan, W.-C. Tetrahedron 2010, 66, 1441.

(d) Hu, S.; Zhang, L.; Li, J.; Luo, S.; Cheng, J.-P. Eur. J. Org. Chem. 2011, 3347.

[12] Pousse, G.; Le Cavelier, F.; Humphreys, L.; Rouden, J.; Blanchet, J. Org. Lett. 2010, 12, 3582.

[13] For examples of soft enolization, see: (a) Rathke, M. W.; Cowan, P. J. J. Org. Chem. 1985, 50, 2622.

(b) Rathke, M. W.; Nowak, M. J. Org. Chem. 1985, 50, 2624.

(c) Tirpak, R. E.; Olsen, R. S.; Rathke, M. W. J. Org. Chem. 1985 , 50,4877 .

(d) Kohler, M. C.; Yost, J. M.; Garnsey, M. R.; Coltart, D. M. Org. Lett. 2010, 12, 3376.

(e) Jiang, X.; Cao, Y.; Wang, Y.; Liu, L.; Shen, F.; Wang, R. J. Am. Chem. Soc. 2010, 132, 15328.

[14] (a) Guo, Q.; Bhanushali, M.; Zhao, C.-G. Angew. Chem., Int. Ed. 2010, 49, 9460 .

(b) Liu, G.-G.; Zhao, H.; Lan, Y.-B.; Wu, B.; Huang, X.-F.; Chen, J.; Tao, J.-C.; Wang, X.-W. Tetrahedron 2012, 68, 3843.

(c) Liu, H.; Wu, H.; Luo, Z.; Shen, J.; Kang, G.; Liu, B.; Wan, Z.; Jiang, J. Chem. Eur. J. 2012, 18, 11899.

For the Henry reaction of $\mathrm{CH}_{3} \mathrm{NO}_{2}$ and isatins, see: (d) Liu, L.; Zhang, S.; Xue, F.; Lou, G.; Zhang, H.; Ma, S.; Duan, W.; Wang, W. Chem. Eur. J. 2011, 17, 7791.

(e) Li, M.-Q.; Zhang, J.-X.; Huang, X.-F.; Wu, B.; Liu, Z.-M.; Chen, J.; Li, X.-D.; Wang, X.-W. Eur. J. Org. Chem. 2011, 5237.

(f) Zhang, Y.; Li, Z.; Xu, H.; Zhang, Y.; Wang, W. RSC Adv. 2011, 1,389 .

[15] Aikawa, K.; Mimura, S.; Numata, Y.; Mikami, K. Eur. J. Org. Chem. 2011, 62.

[16] Hara, N.; Nakamura, S.; Funahashi, Y.; Shibata, N. Adv. Synth. Catal. 2011, 353, 2976.

[17] For a review, see: Liu, Y.-L.; Yu, J.-S.; Zhou, J. Asian J. Org. Chem. 2013, 2, 194.

[18] (a) Liu, Y.-L.; Zhou, J. Chem. Commun. 2012, 48, 1919. For our related work, see; (b) Liu, Y.-L.; Zhou, J. Acta Chim. Sinica 2012, 70, 1451.

[19] Tomita, D.; Yamatsugu, K.; Kanai, M.; Shibasaki, M. J. Am. Chem. Soc. 2009, 131, 6946 .

[20] (a) Iwabuchi, Y.; Nakatani, M.; Yokoyama, N.; Hatakeyama, S. J. Am. Chem. Soc. 1999, 121, 10219.

(b) Iwabuchi, Y.; Furukawa, M.; Esumi, T.; Hatakeyama, S. Chem. Commun. 2001, 2030.

(c) Kawahara, S.; Nakano, A.; Esumi, T.; Iwabuchi, Y.; Hatakeyama, S. Org. Lett. 2003, 5, 3103.

(d) Nakano, A.; Kawahara, S.; Akamatsu, S.; Morokuma, K.; Nakatani, M.; Iwabuchi, Y.; Takahashi, K.; Ishihara, J.; Hatakeyama, S. Tetrahedron 2006, 62, 381 .

(e) Nakano, A.; Takahashi, K.; Ishihara, J.; Hatakeyama, S. Org.
Lett. 2006, 8, 5357.

[21] For reviews, see: (a) Basavaiah, D.; Rao, A. J.; Satyanarayana, T. Chem. Rev. 2003, 103, 811.

(b) Farina, V.; Reeves, J. T.; Senanayake, C. H.; Song, J. J. Chem. Rev. 2006, 106, 2734.

(c) Masson, G.; Housseman, C.; Zhu, J. Angew. Chem., Int. Ed. 2007, 46, 4614.

(d) Shibasaki, M.; Kanai, M. Chem. Rev. 2008, 108, 2853.

(e) Ma, G.-N.; Jiang, J.-J.; Shi, M.; Wei, Y. Chem. Commun. 2009, 5496.

(f) Wei, Y.; Shi, M. Acc. Chem. Res. 2010, 43, 1005.

For a example, see (g) Hyodo, K.; Nakamura, S.; Shibata, N. Angew. Chem., Int. Ed. 2012, 51, 10337.

[22] (a) Liu, Y.-L.; Wang, B.-L.; Cao, J.-J.; Chen, L.; Zhang, Y.-X.; Wang, C.; Zhou, J. J. Am. Chem. Soc. 2010, 132, 15176.

(b) Liu, Y.-L.; Zeng, X.-P.; Zhou, J. Chem.-Asian J. 2012, 7, 1759.

(c) Guan, X.-Y.; Wei, Y.; Shi, M. Chem. Eur. J. 2010, 16, 13617.

(d) Zhong, F.; Chen, G.-Y.; Lu, Y. Org. Lett. 2011, 13, 82.

(e) Wang, C.-C.; Wu, X.-Y. Tetrahedron 2011, 67, 2974.

(f) Qian, J.-Y.; Wang, C.-C.; Sha, F.; Wu, X.-Y. RSC Adv. 2012, 2, 6042 .

(g) Zeng, X.-P.; Liu, Y.-L.; Ji, C.-B.; Zhou, J. Chin. J. Chem. 2012, 30, 2631.

[23] For reviews, see: (a) Denmark, S. E.; Beutner, G. L. Angew. Chem., Int. Ed. 2008, 47, 1560.

(b) Gawronski, J.; Wascinska, N.; Gajewy, J. Chem. Rev. 2008, $108,5227$.

(c) Ooi, T., Maruoka, K. Acc. Chem. Res. 2004, 37, 526.

[24] Kitajima, M.; Mori, I.; Arai, K.; Kogure, N.; Takayama, H. Tetrahedron Lett. 2006, 47, 3199.

[25] Itoh, J.; Han, S. B.; Krische, M. J. Angew. Chem., Int. Ed. 2009, 48, 6313.

[26] (a) Qiao, X.-C.; Zhu, S.-F.; Zhou, Q.-L. Tetrahedron: Asymmetry 2009, 20, 1254.

(b) Zhu, S.-F.; Qiao, X.-C.; Zhang, Y.-Z.; Wang, L.-X.; Zhou, Q.-L. Chem. Sci. 2011, 2, 1135.

[27] Cao, Z.-Y.; Zhang, Y.; Ji, C.-B.; Zhou, J. Org. Lett. 2011, 13, 6398.

[28] Hanhan, N. V.; Tang, Y. C.; Tran, N. T.; Franz, A. K. Org. Lett. 2012, 14, 2218.

[29] Zheng, K.; Yin, C.; Liu, X.; Lin, L.; Feng, X. Angew. Chem., Int. Ed. 2011, 50, 2573.

[30] Sano, D.; Nagata, K.; Itoh, T. Org. Lett. 2008, 10, 1593.

[31] Yang, Y.; Moinodeen, F.; Chin, W.; Ma, T.; Jiang, Z.; Tan, C.-H. Org. Lett. 2012, 12, 4762.

[32] (a) Bui, T.; Candeias, N. R.; Barbas III, C. F. J. Am. Chem. Soc. 2010, 132, 5574.

(b) Liao, Y.-H.; Wu, Z.-J.; Han, W.-Y.; Zhang, X.-M.; Yuan, W.-C. Chem. Eur. J. 2012, 18, 8916.

[33] Du, T.-P.; Zhu, G.-G.; Zhou, J. Lett. Org. Chem. 2012, 9, 225.

[34] (a) Shintani, R.; Inoue, M.; Hayashi, T. Angew. Chem., Int. Ed. 2006, 45,3353 .

(b) Lai, H.; Huang, Z.; Wu, Q.; Qin, Y. J. Org. Chem. 2009, 74, 283.

For other examples, see: (c) Toullec, P. Y.; Jagt, R. B. C.; de Vries, J. G.; Feringa, B. L.; Minnaard, A. J. Org. Lett. 2006, 8, 2715.

(d) Feng, X.; Nie, Y.; Yang, J.; Du, H. Org. Lett. 2012, 14, 624.

(e) Gui, J.; Chen, G.; Cao, P.; Liao, J. Tetrahedron: Asymmetry 2012, 23, 554.

(f) Liu, Z.; Gu, P.; Shi, M.; McDowell, P.; Li, G. Org. Lett. 2011, $13,2314$.

[35] (a) Hanhan, N. V.; Sahin, A. H.; Chang, T. W.; Fettinger, J. C.; Franz, A. K. Angew. Chem., Int. Ed. 2010, 49, 744. 
(b) Gutierrez, E. G.; Wong, C. J.; Sahin, A. H.; Franz, A. K. Org. Lett. 2011, 13, 5754.

[36] Deng, J.; Zhang, S.; Ding, P.; Jiang, H.; Wang, W.; Li, J. Adv. Synth. Catal. 2010, 352, 833.

[37] Chauhan, P.; Chimni, S. S. Chem. Eur. J. 2010, 16, 7709.

[38] Ishimaru, T.; Shibata, N.; Nagai, J.; Nakamura, S.; Toru, T.; Kane- masa, S. J. Am. Chem. Soc. 2006, 128, 16488.

[39] Zhang, Z.; Zheng, W.; Antilla, J. C. Angew. Chem., Int. Ed. 2011, $50,1135$.

[40] Yin, L.; Kanai, M.; Shibasaki, M. Angew. Chem., Int. Ed. 2011, 50, 7620.

(Cheng, F.) 